\title{
Programme zur Methode der Finiten Elemente
}

\section{Working Paper}

Author(s):

Anderheggen, Edoardo

Publication date:

1969

Permanent link:

https://doi.org/10.3929/ethz-a-000747213

Rights / license:

In Copyright - Non-Commercial Use Permitted

Originally published in:

Bericht / Institut für Baustatik ETH Zürich 23 
Programme zur Methode der Finiten Elemente

Edoardo Anderheggen

Bericht Nr. 23 


\section{Programme zur Methode der Finiten Elemente}

von

Dr. sc. techn. Edoardo Anderheggen

Institut für Baustatik

Eidgenössische Technische Hochschule Zürich 
TEIL I : PROGRAMMBESCHREIBUNGEN

2. ELEMENT PROGRAMME : DRPL21, DRPL18, DRSC18, VKPL28, VKPL24, VKSC24

2.1 Anwendungen

2.2 Programmtechnische Einzelheiten

2.3 Die dreieckigen Plattenelemente DRPL21 und DRPL18

2.4 Das dreieckige Scheibenelement DRSC18

2.5 Die viereckigen Elemente VKPL28, VKPL24 und VKSC24

2.6 Elastisch gestutzte viereckige Plattenelemente

3. PROGRAMME ZUR LOESUNG VON SYMMETRISCHEN GLEICHUNGSSYSTEMEN MIT BANDFOERMIGER KOEFFIZENTENMATRIX : BANDMAT, BMRESID

3.1 Anwendungen

3.2 Organisation der Datenubertragung

3.3 Die Subroutine BANDMAT

3.4 Die Subroutine BMRESID

ANHANG I : KONDENSATION DER INTERNEN FREIHEITSGRADE FUER NICHT GESTUETZTE UND FUER ELASTISCH GESTUETZTE VIERECKIGE PLATTENELEMENTE

TEIL II : PROGRAMME

1. Subroutine DRPL21

2. Subroutine DRSC18

3. Subroutine VKPL 28

4. Subroutine VKPL 24

5. Subroutine VKSC24

6. Subroutine BANDMAT

7. Subroutine BMRESID 
1. EINLEITUNG

Zweck dieses Berichtes ist die Veröffentlichung einiger Computerprogramme, welche zur elastischen Analyse von Flächentragwerken nach der Methode der finiten Elemente benützt werden können. Diese wurden am Institut für Baustatik, Abteilung Massivbau der Eidgenössischen Technischen Hochschule Zürich im Rahmen eines entsprechenden Forschungsprogrammes entwickelt.

Die ersten sechs Programme (DRPL21, DRPL18, DRSC18, VKPL28, VKPL24 und VKSC24) berechnen die Steifigkeitsmatrizen und verschiedene Lastvektoren für dreieckige und viereckige Platten- und Scheibenelemente. Die zwei letzten (BANDMAT und BMRESID) dienen zur Lösung von grossen symmetrischen Gleichungssystemen, deren Koeffizentenmatrix eine Bandstruktur aufweist. Die Programme sind in Form von FORTRAN IV Subroutinen geschrieben und wurden auf dem Computer Control-Data 6500 der Firma "FIDES" in Zürich vìa ETH-Satellit getestet.

Der erste Teil dieses Berichtes erk1ärt die Funktion jeder Subroutine und schildert wie dieselben in ein Hauptprogramm einzubauen sind. Es wird dabei angenommen, dass der Leser sowohl mit der Programmiersprache FORTRAN IV wie mit der Matrizenrechnung und der Methode der finiten Elemente vertraut ist. Man wird hingegen auf eine detaillierte Herleitung der Theorie, auf welche die Programme aufgebaut sind, nicht eingehen, weil diese in der Fachliteratur an verschiedenen Stellen zu finden ist.

Der zweite Teil dieses Berichtes enthält die Programme selber. Diese sollten, dank der vielen "Comment"-Karten, die Arbeitsweise der verschiedenen Subroutinen verständlich machen. Die entsprechenden Karten können vom Institut für Baustatik, Abteilung Massivbau der ETH Zürich verlangt werden. 


\section{$\underline{2.1}$ Anwendungen}

Diese Programme bestimmen die Steifigkeitsmatrizen sowie verschiedene Lastvektoren für dreieckige und viereckige Platten- und Scheibenelemente mit orthotropischen elastischen Eigenschaften. Bei den viereckigen Elementen werden auch die Matrizen und Vektoren bestimmt, welche zur Berechnung der Verschiebungen und der Spannungen im Schwerpunkt des Elementes notwendig sind. Der Fall von elastisch gestützten viereckigen Plattenelementen ist ebenfalls behandelt.

Die verschiedenen Matrizen und Vektoren werden auf Grund von polynominalen Verschiebungsansätzen bestimmt. Sämtliche kinematischen Bedingungen, die für die Konvergenz der Resultate bei Verfeinerung der Elementeinteilung notwendig sind, werden dabei erfullt.

Das wesentliche Merkmal dieser Elemente besteht darin, dass nicht nur Verschiebungen, sondern auch Krümmungen (bei den Plattenelementen) oder Dehnungen (bei den Scheibenelementen) als Verschiebungsvariablen auftreten. Dies hat zur Folge, dass die Spannungsberechnungen in jeder Ecke der Elemente trivial werden. Besitzen alle Elemente, die an einem Knoten miteinander verbunden sind, die gleichen elastischen Steifigkeitskoeffizenten, wird der Spannungsverlauf über diesem Knoten kontinuierlich sein. Dabei wird selbst mit einer groben Elementeinteilung, dank den verwendeten hochgradigen Verschiebungsansätzen, eine hohe Genauigkeit erreicht. Die hier angeführten Elemente dürften deswegen vor allem für genaue elastische Spannungsberechnungen nach der Theorie 1. Ordnung geeignet sein. Alle Arten von F1ächentragwerken lassen sich damit behandeln.

Numerische Resultate sowie weitere Angaben über diese Elemente sind in der Literatur an den angegebenen Stellen zu finden.

\subsection{Programmtechnische Einzelheiten}

Die Namen der "Entry Points" der FORTRAN-Subroutinen wurden so gewählt, dass die verschiedenen Fälle sich unmittelbar erkennen 1assen. Zum Beispiel:

DRPL21 : DReieckiges PLattenelement mit 21 Verschiebungsvariablen

VKSC24 : Viereckiges SCheibenelement mit $\underline{24}$ Verschiebungsvariablen

Die Uebertragung der Daten zwischen dem Hauptprogramm und diesen Element-Subroutinen ist wie folgt organisiert:

1. Die formalen Parameter entsprechen den Resultaten der Subroutine. Ihre Bedeutung wird jeweils im Programm selbst, mit Hilfe von "Comment"-Karten, erkIart.

2. Die Ausgangsdaten werden via "Labeled Common" übertragen. Die Namen dieser "Labeled Commons" sind, den Namen der Subroutinen entsprechend: CDRPL, CDRSC, CVKPL und CVKSC.

3. Die beiden "Labeled Commons" SCR1 und SCR2 sind Arbeitszonen (oder "SCRatch"Zonen) des Speichers und werden zur Datenübertragung nicht verwendet. Sie können, um Platz in Kernspeichern zu sparen, in anderen Programmteilen wieder als Arbeitszonen benützt werden. 
4. Nur die obere Halfte der symmetrischen Steifigkeitsmatrix [K] $]_{n \times n}$ wird bestimmt, und, beginnend in der Adresse <STEIFM> zeilenweise gespeichert $(n \cdot(n+1) / 2$ Koeffizenten).

Selbstverständlich ist es möglich, mit wenigen und übersichtlichen Programmänderungen die Datenübertragung auf andere Weise zu gestalten.

\section{$\underline{2.3}$ Die dreieckigen P1attenelemente DRPL21 und DRPL18 (s. Lit. ${ }^{1}, 2,3,4,5$ )}

Ein vollständiges Polynom 5. Grades in $x$ und $y$ wird als Durchbiegungsfunktion $w(x, y)$ angenommen. Da ein solches Polynom von 21 Parametern bestimmt wird, muss das entsprechende Plattenelement 21 diskrete Verschiebungsvariablen besitzen. Diese bilden zusammen einen Verschiebungsvektor $\{w\} 21 \times 1$, der bei dem dreieckigen Plattenelement DRPL21 wie folgt definiert ist (s. Fig. 1.):

$$
\begin{aligned}
& \{w\}^{T} \equiv<w_{1} \quad w_{1, x} \quad w_{1, y} \quad w_{1, x x} \quad w_{1, y y} \quad w_{1, x y} \\
& \mathrm{w}_{2} \quad \mathrm{w}_{2, x} \quad \mathrm{w}_{2, y} \quad \mathrm{w}_{2, x x} \quad \mathrm{w}_{2, y y} \quad \mathrm{w}_{2, x y} \\
& w_{3} \quad w_{3, x} \quad w_{3, y} \quad w_{3, x x} \quad w_{3, y y} \quad w_{3, x y} \\
& -\mathrm{w}_{12, \mathrm{n}} \quad \mathrm{w}_{23, \mathrm{n}} \quad \mathrm{w}_{31, \mathrm{n}}>\text { (*) }
\end{aligned}
$$

Die Numern 1, 2, 3 sind die Eckpunktnummern, numeriert im Gegenuhrzeigersinn. Die drei letzten Verschiebungsvariablen sind die Ableitungen der Durchbiegungsfunktion oder die Rotation in Normalrichtung in der Mitte jeder Seite. Diese werden beim Element DRPL18 (**) eliminiert, indem die Bedingung gestellt wird, dass die Ableitung der Durchbiegungsfunktion in Normalrichtung entlang jeder Seite eine Funktion 3 . Grades ist (und nicht 4. Grades wie beim Element DRPL21). Die entsprechenden Freiheitsgrade gehen dabei verloren. Der Verschiebungsvektor reduziert sich von ${ }^{[w\}}{ }_{21 \times 1}$ auf ${ }^{\{w}{ }_{18 \times 1}$.

Die angegebene Wahl der Verschiebungsvariablen garantiert die Erfullung der kinematischen Kompatibilitätsbedingungen zwischen Elementen. Bei der Bildung der globalen Steifigkeitsmatrix mit Plattenelementen, die Seitenfreiheitsgrade besitzen (d.h. DRPL21 und VKPL28: s. Abschnitt 2.5), muss auf die Vorzeichen dieser Variablen geachtet werden. Damit sie der gleichen Rotation entsprechen, müssen die Vorzeichen der Seitenvariablen entgegengesetzt sein. Die Vorzeichenkonvention ist folgende: eine positive Rotation $+w$ erzeugt positive Durchbiegungen im Innern des Elementes. Zur Definition der Steifigkeitsmatrix $[\mathrm{K}]_{21 \times 21}$ (DRPL21) bzw. [K] ${ }_{18 \times 18}$ (DRPL18) gilt folgende Beziehung:

$$
U=\frac{1}{2} \iint\left(w_{, x x} M_{x}+w_{, y y} M_{y}+2 w, x y M_{x y}\right) d F=\frac{1}{2}\{w\}^{T}[K]\{w\}
$$

(*) Ein Komma gefolgt von einem oder mehreren Indices bedeutet partielle Ableitung nach der entsprechenden Richtung. Zum Beispiel:

$$
{ }_{,}, x \equiv \frac{\partial w}{\partial x} \quad w, x y \equiv \frac{\partial^{2} w}{\partial x \partial y} \quad \text { usw. }
$$

(**) DRPL18 ist ein "Entry Point" der Subroutine DRPL21. 
Dabei ist $U$ die elastische Formänderungsarbeit des Plattenelementes (mit F1äche F) für einen vom Vektor $\{w\}$ spezifizierten Verschiebungszustand. $M_{x}, M_{y}$ und $M_{x y}$ sind die beiden Biegemomente und das Drillungsmoment. Diese werden aus den Krümmungen ${ }^{w}, x x,{ }^{,}, y y$ und $w, x y$ mit Hilfe der Steifigkeitskoeffizenten $d_{11}, d_{12}, d_{22}$ und $d_{33}$ bestimmt. Zur Definition dieser Koeffizenten gelten folgende Beziehungen:

$$
\begin{aligned}
& M_{x}=d_{11} w_{, x x}+d_{12} w, y y \\
& M_{y}=d_{12}{ }^{w}, x x+d_{22}{ }^{,}, y y \\
& M_{x y}=d_{33}{ }^{w}, x y
\end{aligned}
$$

Für isotrope Platten gilt:

$$
\begin{aligned}
& d_{11}=d_{22}=\frac{E \cdot t^{3}}{12\left(1-v^{2}\right)} \\
& d_{12}=v \cdot d_{11} \\
& d_{33}=(1-v) \cdot d_{11}
\end{aligned}
$$

Wobei E der Elastizitätsmodul, $t$ die konstante Plattendicke und $v$ der Poisson'sche Koeffizent sind.

Der Lastvektor $\{P\}_{21 \times 1}$ bzw. $\{P\}_{18 \times 1}$ für eine auf der Fläche des Elementes gleichmässig verteilten Belastung $p=1$ wird ebenfalls bestimmt. Zur Definition dieses Vektors gilt folgende Beziehung:

$$
\mathrm{V}=\iint \mathrm{p} w \mathrm{dF}=\mathrm{p} \cdot \iint \mathrm{w} d \mathrm{~F}=\left\{\mathrm{w}^{\mathrm{T}}\{\mathrm{P}\} \cdot \mathrm{p}\right.
$$

Dabei ist $V$ die ăussere Arbeit der gleichmässig verteilten Last $p$ für einen vom Vektor $\{w\}$ spezifizierten Verschiebungszustand des Plattenelementes. Der Vektor $\{P\}_{21 \times 1} b z w$. $\left.{ }^{\{P}\right\}_{18 \times 1}$ wird, beginnend in der Adresse <GLEICHP>, sequentiell gespeichert.

Die Geometrie des Elementes wird von den Koordinaten $\left(x_{1}, y_{1}\right)$ und $\left(x_{2}, y_{2}\right)$ der Knotenpunkte 1 und 2 relativ $z u 3$ bestimmt. Die orthotropischen Steifigkeitskoeffizenten sind im Innern des Plattenelementes konstant.

Der Algorithmus der Subroutine DRPL21 (wie auch der des Scheibenelementes DRSC18) benötigt keine Matrixinversion und keine explizite Integrationsprozedur. Dies wird erreicht durch die Anwendung von natürlichen, dimensionslosen Dreieckskoordinaten. Da die in diesem Koordinatensystem hergeleiteten Matrizen und Vektoren von der Geometrie und von den elastischen Eigenschaften des Elementes weitgehend unabhängig werden, ist es möglich, viele Berechnungen nur einmal für alle Elemente durchzuführen. Die entsprechenden Daten werden dann als Konstante (mittels "DATA-Statements") im Computer gespeichert, so dass die Bestimmung der Steifigkeitsmatrizen und der Lastvektoren in globalen kartesischen Koordinaten nur noch einfache Transformationen verlangt. Solche Prozeduren sind heute allgemein bekannt und verkürzen die notwendige Computerzeit erheblich.

\section{$\underline{2.4 \text { Das dreieckige Scheibenelement DRSC18 }}$ (s. Lit. $5,6,7$ )}

Zwei vollständige Polynome 3. Grades in $x$ und $y$ werden als Verschiebungsfunktionen $u(x, y)$ und $v(x, y)$ in $x$ - und $y$-Richtung angenommen. Da jedes von diesen Polynomen von 10 Parametern bestimmt wird, muss das entsprechende dreieckige Scheibenelement 20 diskrete Verschiebungsvariablen besitzen. Diese bilden $z$ wei Vektoren $\left\{w_{e}\right\}_{18 \times 1}$ und $\left\{w_{i}\right\}_{2 \times 1}$ 


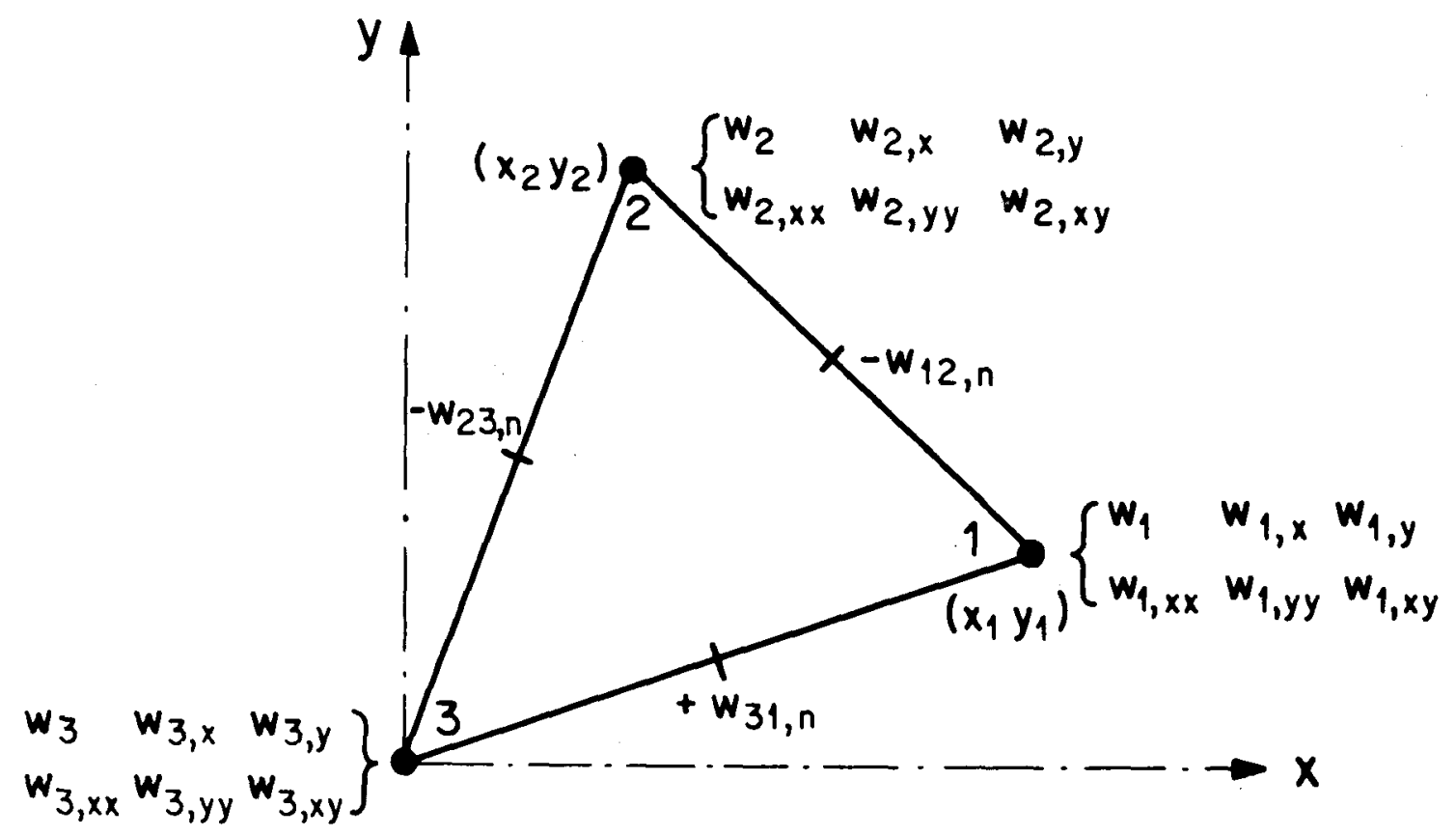

Fig. 1: Plattenelement DRPL21 mit 21 Verschiebungsvariablen

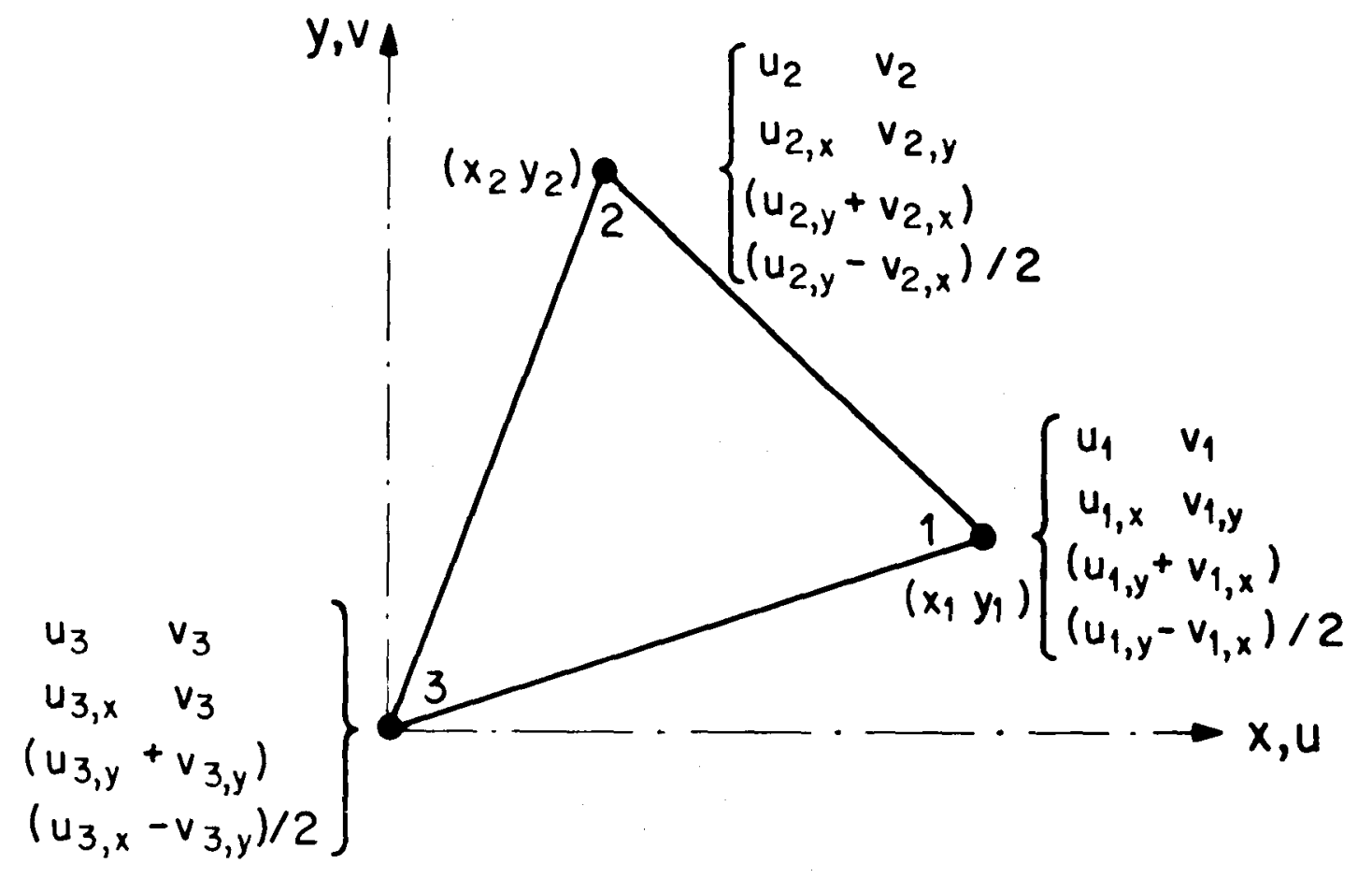

Fig. 2: Scheibenelement DRSC18 mit 18 Verschiebungsvariablen 
die wie folgt definiert sind (s. Fig. 2):

$$
\begin{aligned}
& \left\{w_{e}\right\}^{T} \equiv<u_{1} \quad v_{1} \quad u_{1, x} \quad v_{1, y} \quad\left(u_{1, y}+v_{1, x}\right) \quad\left(u_{1, y}-v_{1, x}\right) / 2 \\
& u_{2} \quad v_{2} \quad u_{2, x} \quad v_{2, y} \quad\left(u_{2, y}+v_{2, x}\right) \quad\left(u_{2, y}-v_{2, x}\right) / 2 \\
& u_{3} \quad v_{3} \quad u_{3, x} \quad v_{3, y} \quad\left(u_{3, y}+v_{3, x}\right) \quad\left(u_{3, y}-v_{3, x}\right) / 2> \\
& \left\{w_{i}\right\}^{T} \equiv<\iint u d F \quad \iint v d F>
\end{aligned}
$$

Dabei sind $u_{n, x}=\varepsilon_{x}, u_{n, y}=\varepsilon_{y}$ und $u_{n, y}+v_{n, x}=r$ die beiden Dehnungen und die Schiebung am Eckpunkt $n(n=1,2,3)$. Die Variable $\left(u_{n, y}-v_{n, x}\right) / 2=\omega$ kann als Rotation des Eckpunktes $n$ um eine zur Scheibenebene senkrecht stehenden Achse aufgefasst werden $(*)$.

Die Verschiebungsvariablen des Vektors $\left\{w_{e}\right\}_{18 \times}$ bestimmen die Verschiebungen der Elementränder und werden deswegen als "externe" Variablen bezeichnet. Sie sind so gewäh1t, dass die kinematischen Kompatibilitätsbedingungen zwischen Elementen erfü11t werden. Die beiden "internen" Variablen des Vektors $\left\{w_{i}\right\}$ beeinflussen hingegen die Randverschiebungen nicht und können somit willkürlich gewăhlt werden. Die hier getroffene Wahl scheint günstiger zu sein als die Wahl der Schwerpunktverschiebungen $u$ und $v$ (s. Lit. ${ }^{5}, 6,7$ ). Insbesondere wird damit die Bestimmung der Lastvektoren für gleichmässig verteilte Massenkräfte in u- und v-Richtung wesentlich erleichtert.

Bekanntlich ist es möglich, durch die Anwendung des Minimumprinzips der potentiellen Energie im Innern des Elementes die interne Verschiebungsvariablen zu eliminieren oder zu "kondensieren". Dadurch geht kein Freiheitsgrad verloren. Diese Prozedur wird im Anhang I kurz erklärt.

Zur Definition der "kondensierten" Steifigkeitsmatrix $[\mathrm{K}]_{18 \times 18}$ gilt folgende Beziehung :

$$
U=\frac{1}{2} \iint\left(\varepsilon_{x} N_{x}+\varepsilon_{y} N_{y}+\gamma N_{x y}\right) d F=\frac{1}{2}\left\{w_{e}\right\}^{T}[K]\left\{w_{e}\right\}
$$

Dabei ist $U$ die elastische Formänderungsarbeit des Scheibenelementes für einen vom Vektor $\left\{w_{e}\right\}$ spezifizierten Verschiebungszustand. $\mathrm{N}_{x}, \mathrm{~N}_{y}$ und $\mathrm{N}_{x y}$ sind die Normal- und Schubkräfte pro Längeneinheit, die in der Scheibe wirken. Diese werden aus den Dehnungen $u_{, x}=\varepsilon_{x}, v_{, y}=\varepsilon_{y}$ und aus der Schiebung $u_{, y}+v_{, x}=\gamma$ mit Hilfe der orthotropischen Steifigkeitskoeffizenten $d_{11}, d_{12}, d_{22}$ und $d_{33}$ bestimmt. Zur Definition dieser Koeffizenten gelten folgende Beziehungen:

$$
\begin{aligned}
& N_{x}=d_{11} u_{, x}+d_{22} v_{, y}=d_{11} \varepsilon_{x}+d_{12} \varepsilon_{y} \\
& N_{y}=d_{12} u_{, x}+d_{22} v_{, y}=d_{12} \varepsilon_{x}+d_{22} \varepsilon_{y} \\
& N_{x y}=d_{33}\left(u_{, y}+v_{, x}\right)=d_{33} \gamma
\end{aligned}
$$

(*) Diese Rotations-Variable w erlaubt bei Schalenproblemen eine konsistente Definition des räumlichen Verschiebungsvektors für jeden Knotenpunkt. 
Für isotrope Scheiben gilt:

Für ebene Spannungsprobleme $\left(\sigma_{z}=0, \varepsilon_{z} \neq 0\right)$ :

$$
\begin{aligned}
& \mathrm{d}_{11}=\mathrm{d}_{22}=\mathrm{E} \cdot \mathrm{t} \\
& \mathrm{d}_{12}=\mathrm{v} \cdot \mathrm{d}_{11}=\mathrm{v} \cdot \mathrm{E} \cdot \mathrm{t} \\
& \mathrm{d}_{33}=\mathrm{d}_{11} / 2(1+v)=\mathrm{G} \cdot \mathrm{t}
\end{aligned}
$$

Für ebene Dehnungsprobleme $\left(\varepsilon_{z}=0, \sigma_{z} \not 0\right)$ :

$$
\begin{aligned}
& \mathrm{d}_{11}=\mathrm{d}_{12}=\frac{\mathrm{E} \cdot \mathrm{t} \cdot(1-v)}{(1+v)(1-2 v)} \\
& \mathrm{d}_{12}=\frac{v \cdot E \cdot t}{(1+v)(1-2 v)} \\
& \mathrm{d}_{33}=\frac{E \cdot t}{2(1+v)}=G \cdot t
\end{aligned}
$$

wobei $t$ die konstante Scheibendicke, E der Elastizitätsmodul, G der Schubmodul und $v$ der Poisson'sche Koeffizent sind.

Die Subroutine DRSC18 bestimmt noch die Lastvektoren $\left\{P_{u}\right\}_{18 \times 1}$ und $\left\{P_{v}\right\}_{18 \times 1}$ für gleichmässig verteilte Massenkräfte pro Flächeneinheit $p_{u}=1$ bzw. $p_{v}=1$ in $u$ - und v-Richtung. Zur Definition dieser beiden Vektoren gelten folgende Beziehungen:

$$
\begin{aligned}
& \mathrm{v}=\iint \mathrm{p}_{\mathrm{u}} \mathrm{u} d F=\mathrm{p}_{\mathrm{u}} \cdot \iint \mathrm{u} d F=\left\{\mathrm{w}_{\mathrm{e}}\right\}^{\mathrm{T}}\left\{\mathrm{P}_{\mathrm{u}}\right\} \cdot \mathrm{p}_{\mathrm{u}} \\
& \text { bzw. } \\
& \mathrm{v}=\iint \mathrm{p}_{\mathrm{v}} \mathrm{v} d F=\mathrm{p}_{\mathrm{v}} \cdot \iint \mathrm{v} d F=\left\{\mathrm{w}_{\mathrm{e}}\right\}{ }^{\mathrm{T}}\left\{\mathrm{P}_{\mathrm{v}}\right\} \cdot \mathrm{p}_{\mathrm{v}}
\end{aligned}
$$

Dabei ist $V$ die Arbeit der äusseren Lasten $p_{u}$ bzw. $p_{v}$ für einen, vor Vektor $\left\{W_{e}\right\}$ spezifizierten Verschiebungszustand des Scheibenelementes. Die Vektoren $\left\{P_{u}\right\}_{18 \times 1}$ und $\left\{P_{v}\right\}_{18 \times 1}$ werden, beginnend in der Adresse <GLEICHU> bzw. <GLEICHV> sequentiell gespeichert.

\subsection{Die viereckigen E1emente VKPL28, VKPL24 und VKSC24}

Viereckige Elemente setzen sich aus vier entsprechenden dreieckigen Elementen zusammen (s. Fig. 3 und 4). Bei den Plattenelementen VKPL28 und VKPL24 handelt es sich um vier DRPL21 Elemente. Die vier externen Seitenvariablen werden bei dem Element VKPL 28 mit der gleichen Prozedur wie beim Element DRPL18 eliminiert.

Bei allen viereckigen Elementen entstehen "interne" Verschiebungsvariablen: 10 für die P1attenelemente VKPL26 oder VKPL24, 6 für das Scheibenelement VKSC24. Diese "internen" Variablen werden "kondensiert". Wie schon erwähnt, wird diese Kondensationsprozedur in Anhang I kurz erklärt.

Die Anordnung der externen Verschiebungsvariablen ist am Anfang der Subroutinen VKPL28, VKPL24 und VKSC24 angegeben. Sie entspricht der Numerierung der Knotenpunkte 


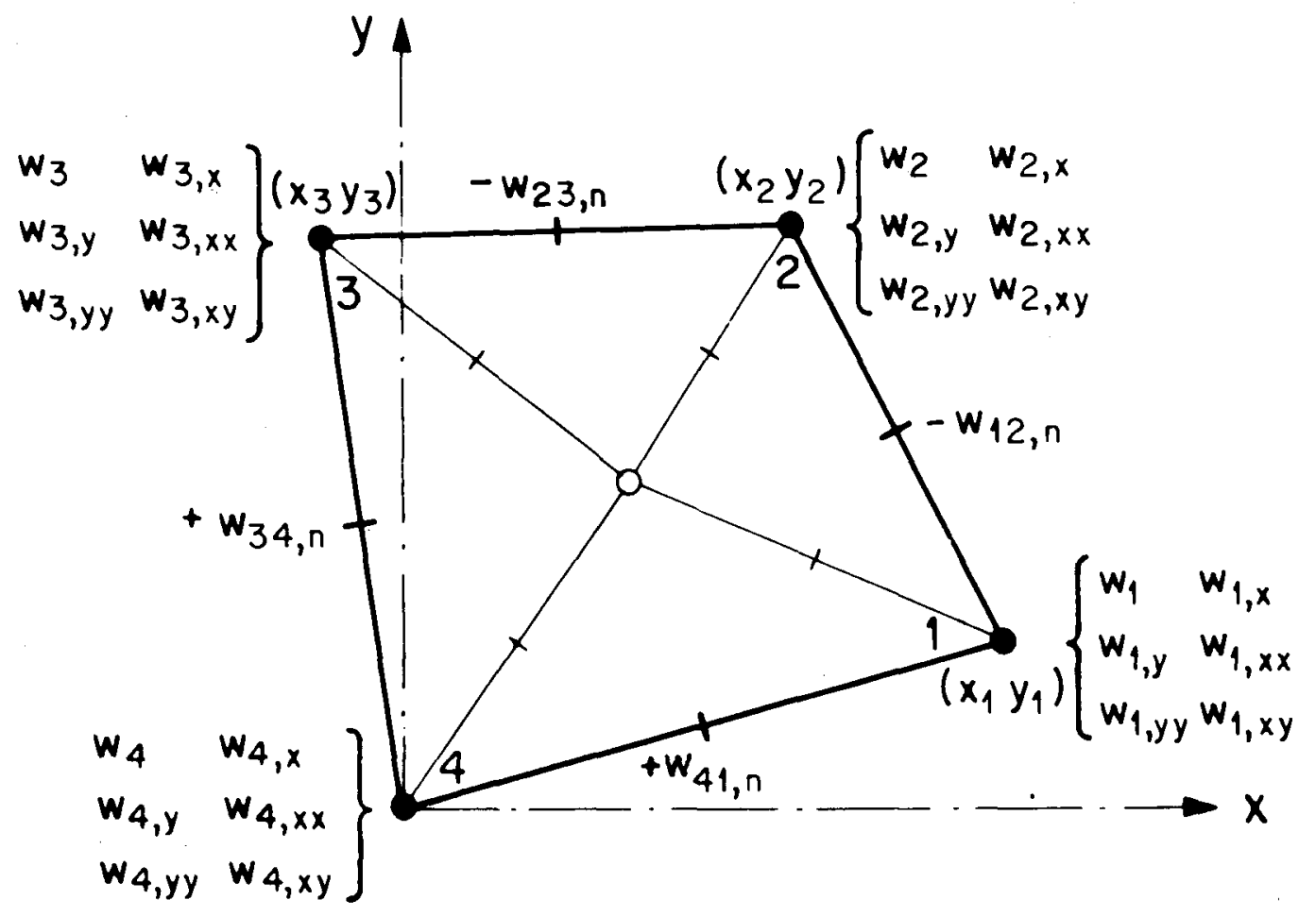

Fig. 3: Plattenelement VKPL28 bestehend aus 4 DRPL21 - Elementen

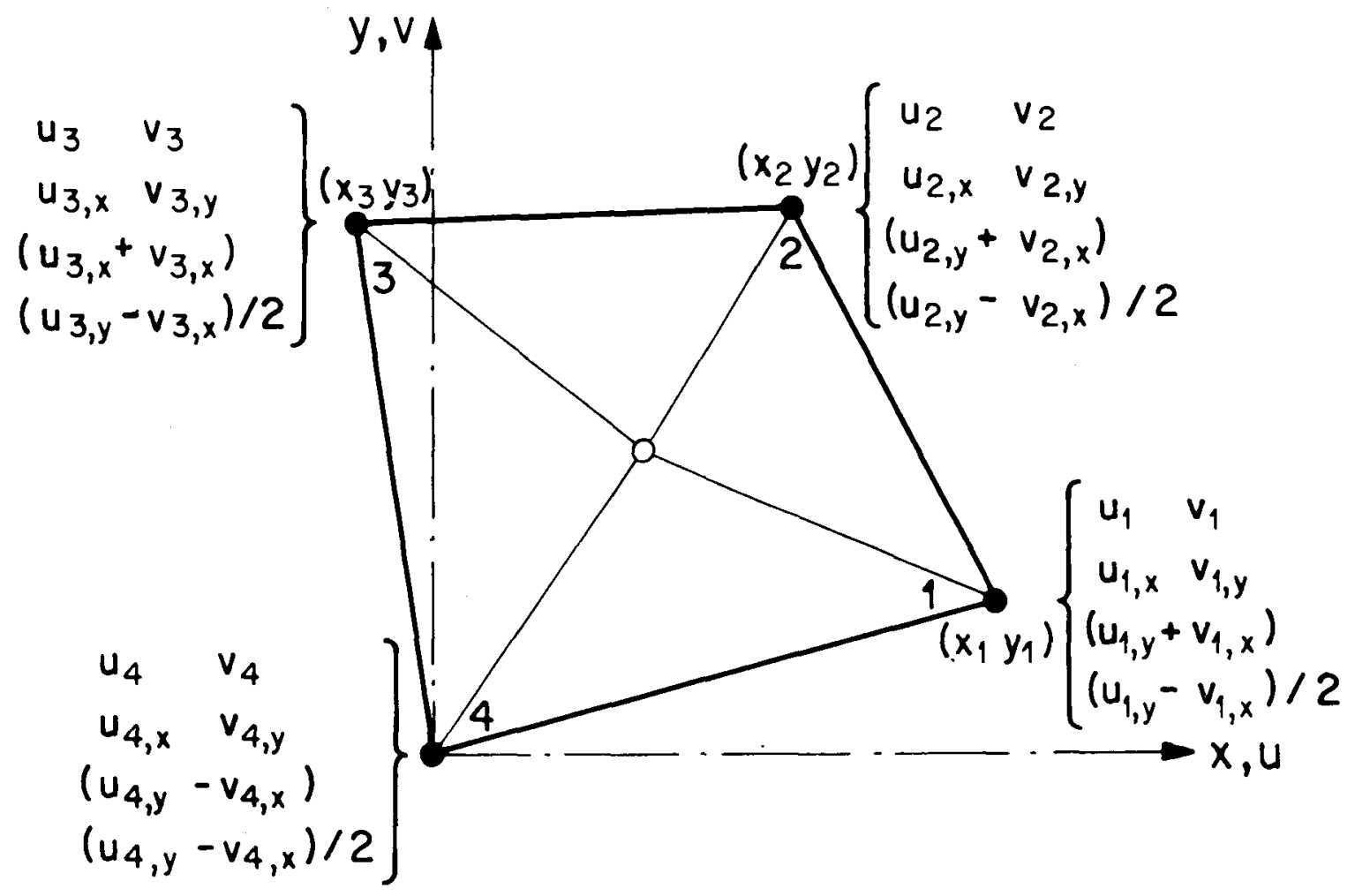

Fig. 4: Scheibenelement VKSC24 bestehend aus 4 DRSC18 - Elementen 
nicht, sollte aber bei der Zusammensetzung der globalen Steifigkeitsmatrix gewisse Vorteile mit sich bringen.

Für die Steifigkeitsmatrizen und die Lastvektoren gelten ähnliche Definitionen wie bei den entsprechenden Dreieckselementen. Bei den P1attenelementen wird noch der Lastvektor für eine im Schwerpunkt des Elementes angreifende Einzellast $P=1$ bestimmt. Dieser Lastvektor wird, beginnend in der Adresse <EINZELP>, sequentiell gespeichert.

Die Subroutinen VKPL28, VKPL24 und VKSC24 bestimmen auch noch die Matrizen und Vektoren, welche zur Berechnung der Verschiebungen und der Spannungen in der Mitte des Elementes notwendig sind. Für die plattenelemente VKPL 28 und VKPL24 gelten folgende Beziehungen:

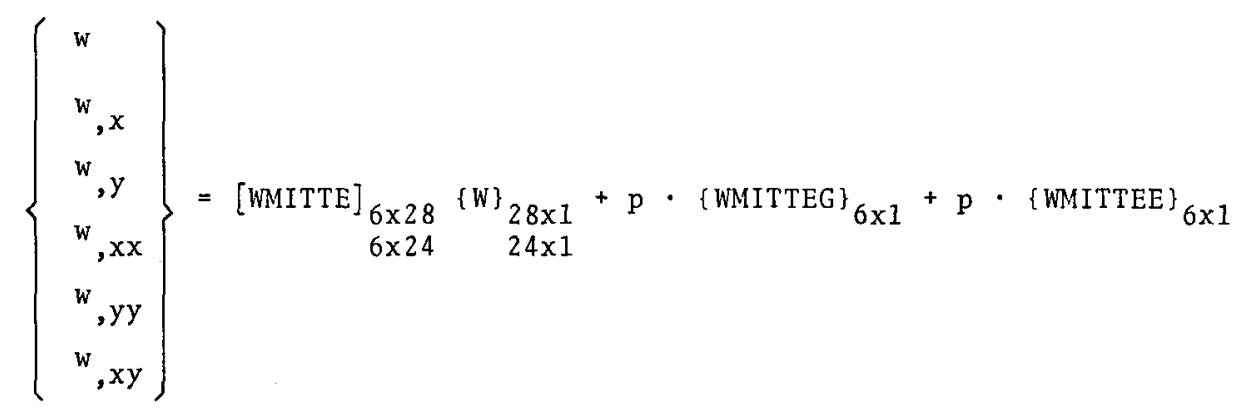

Die Namen der Matrix [WMITTE] und der Vektoren [WMITTEG\} und [WMITTEE\} entsprechen den Namen der zugehörigen formalen Parameter der Subroutinen. \{W\} ist der aus der Lösung des globalen Gleichungsystems erhaltene Verschiebungsvektor. Die skalaren Grössen $\mathrm{p}$ und $\mathrm{P}$ stellen die gleichmässig verteilte Belastung und die in der Mitte des Elementes angreifende Einzellast dar.

Für das Scheibenelement VKSC24 gilt:

$$
\left\{\begin{array}{c}
u \\
v \\
u, x \\
v, y \\
\begin{array}{c}
u, y+v, x \\
(u, y-v, x) / 2
\end{array}
\end{array}\right\}=[\text { WMITTE }]_{6 \times 24}\{W\}_{24 x 1}+p_{u} \cdot\left\{\text { WMITTEU }{ }_{6 x 1}+p_{v} \cdot\{\text { WMITTEV }\} 6 \times 1\right.
$$

Die Namen der Matrix [WMITTE] und der Vektoren \{WMITTEU\} und \{WMITTEV\} entsprechen ebenfa11s den Namen der zugehorigen formalen Parametern. $p_{u}$ und $p_{v}$ sind die gleichmässig verteilten Massenkräfte pro Flächeneinheit in u- und v-Richtung.

Am Anfang der Subroutinen VKPL 28, VKPL24 und VKSC24 wird die "Array" M(252) bzw. M(207) mit Hilfe von "DATA-Statements" initialisiert. Es soll dabei beachtet werden, dass in jedem Computerwort $M(I)$ vier ganzzahlige Zahlen zusammengepackt sind. Jede davon benötigt 12 "Bits", so dass pro Wort 48 "Bits" beansprucht werden. Entsprechende Programmänderungen sind deswegen notwendig, falls diese Subroutinen für einen Computer mit weniger als 48 "Bits" pro Wort oder mit einer anderen Zahlendarste1lung umgeschrieben werden sol1ten. 


\section{$\underline{2.6 \text { Elastisch gestützte viereckige Plattenelemente }}$}

Bei den viereckigen Plattenelementen VKPL28 und VKPL24 wird noch der Fa11 behande1t, bei dem die Elemente von einer elastisch senkbaren Unterlage gestützt werden. Dies erlaubt eine sehr einfache Behandlung des Falles von Platten, die auf frei drehbaren, elastisch senkbaren (oder auch unsenkbaren) Stützen liegen $\left({ }^{*}\right)$. Der entsprechende Algorithmus wird in Anhang I beschrieben. Nach unseren Kenntnissen wurde dieser Algorithmus noch nie verwendet.

Es wird angenommen, dass die Stütze einen, auf die Fläche des Elementes gleichmässig verteilten Druck s ausübt. Die durchschnittliche Einsenkung des Stützenḳopfes \& soll dann proportional zum Stützendruck s und folglich zur stützenkraft $S=F$. s sein. Dies schreibt sich wie folgt:

$$
\delta=\frac{1}{\mathrm{~F}} \iint \mathrm{W} \mathrm{dF}=\mathrm{C} \cdot \mathrm{F} \cdot \mathrm{s}=\mathrm{C} \cdot \mathrm{S}
$$

Dabei ist $C$ die Stützenkonstante, die in den Subroutinen VKPL 28 und VKPL 24 ins "Labeled Common" CVKPL an der Adresse <STKONST> gespeichert wird. C stellt die Einsenkung des Stützenkopfes infolge einer einheitlichen Stützenkraft $S=1$ dar. C darf keinen negativen Wert annehmen, darf aber Null sein (für frei drehbare unsenkbare Stützen). Wird jedoch in der Adresse <STKONST> eine negative Zahl gespeichert, wird das Plattenelement als nicht-gestützt betrachtet (als ob C unendiich gross wäre).

Zur Berechnung der Stützenkraft $S$ aus dem bekannten Lösungsvektor $\{W\}_{28 \times 1}$ bzw. [W\} $_{24 \times 1}$, werden noch der Vektor \{STKRAFT\} $_{28 \times 1}$ bzw. \{STKRAFT\} $24 \times 1$ und die beiden skalaren Grössen STKRG und STKRE bestimmt. Die Stütenkraft S wird dann wie folgt berechnet:

$$
S=\{\text { STKRAFT }\}_{\substack{1 \times 28 \\ 1 \times 24}}^{T} \underset{1 \times 28}{\left[W{ }_{1 \times 24}\right.}+p \cdot S T K R G+P \cdot S T K R E
$$

Die Namen STKRAFT, STKRG und STKRE sind formale Parameter der Subroutinen. $p$ und $P$ sind die früher definierten Plattenbelastungen.

(*) Auch der Fall von Fundamentplatten, die auf einem elastisch senkbaren Boden 1iegen (Bettungsziffertheorie), kann damit behandelt werden. 
KOEFFIZENTENMATRIX : BANDMAT, BMRESID

\subsection{Anwendungen}

Die Methode der endlichen Elemente führt zu grossen linearen Gleichungssystemen, deren Koeffizentenmatrix in vielen Fällen eine Bandstruktur aufweist. Die Subroutinen BANDMAT und BMRESID wurden deswegen speziell für solche bandförmige Matrizen entwikkelt und sind theoretisch im Stande unbeschränkt grosse Gleichungssysteme zu behandeln.

Man geht von folgendem Gleichungssystem aus:

$$
[A]_{N G L x N G L}[X]_{N G L x N B}+[B]_{N G L x N B}=0
$$

wobei $[A]_{N G L x N G L}$ die symmetrische, bandförmige Koeffizentenmatrix (NGL = Anzahl Gleicungen), $[X]_{N G L x N B}$ die Matrix der unbekannten Lösungsvektoren (NB = Anzah1 Belastungsfälle) und $[B]_{N G L x N B}$ die Matrix der Belastungsvektoren ist.

Die Subroutine BANDMAT löst das Gleichungssystem nach dem Gauss'schen Eliminationsverfahren und bestimmt die dreieckige halbinverse Matrix [D] der Koeffizentenmatrix [A] - Die Subroutine BMRESID berechnet die Matrix der Residuenvektoren [R] sowie die Längen derselben dividiert durch die Längen der entsprechenden Belastungsvektoren.

\section{$\underline{3.2 \text { Organisation der Datenübertragung }}$}

Die Datenübertragung erfolgt auf zwei Arten:

1. Durch die formalen Parameter, deren Bedeutung im Programm selbst mit Hilfe von "Comment"-Karten erklärt wird.

2. Durch Anrufe der Lese- und Schreibsubroutinen READMS und WRITMS, welche zur Uebertragung der eigentlichen Matrizen verwendet werden.

Die Matrizen $[A]_{N G L x N G L},[B]_{N G L x N B},[X]_{N G L x N B},[D]_{N G L x N G L}$ und $[R]_{N G L x N B}$ sind in der Regel zu gross, um Platz im Hauptspeicher zu finden. Sie werden deswegen in Blöcke unterteilt und mit Hilfe eines Grossraumspeichers verarbeitet. Fig. 5 zeigt, wie die Koeffizentenmatrix [A], die Lösungsmatrix [X] und die Belastungsmatrix [B] in Blöcke zu zerlegen sind.

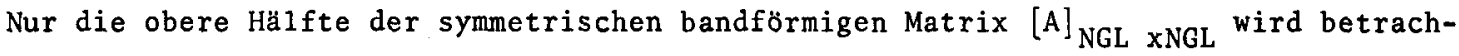
tet. Diese wird in mehrere gleich grosse Blöcke mit je NZ Zeilen und NK Kolonnen unterteilt. Innerhalb dieser Blöcke muss für jeden Koeffizenten (inklusive Nul1-Koeffizent) eine Speicherstelle reserviert werden. Die letzten Blöcke haben ebenfalls dieselben Dimensionen, obwohl sie zum Teil irrelevante Werte enthalten. Es müssen mindestens zwei Blöcke vorhanden sein (NGL > NZ).

Die Matrizen $[B]_{N G L x N B}$ und $[X]_{N G L x N B}$ werden in ähnlicher Weise in gleich grosse B1öcke mit je NZ Zeilen und NB Kolonnen unterteilt. Die Blockunterteilung der Matrizen $[D]_{\text {NGLxNGL }}$ und $[R]_{\text {NGLxNB }}$ entspricht der von $[A]_{\text {NGLxNGL }}$ bzw. $[B]_{\text {NGLxNB }}$ oder 
II

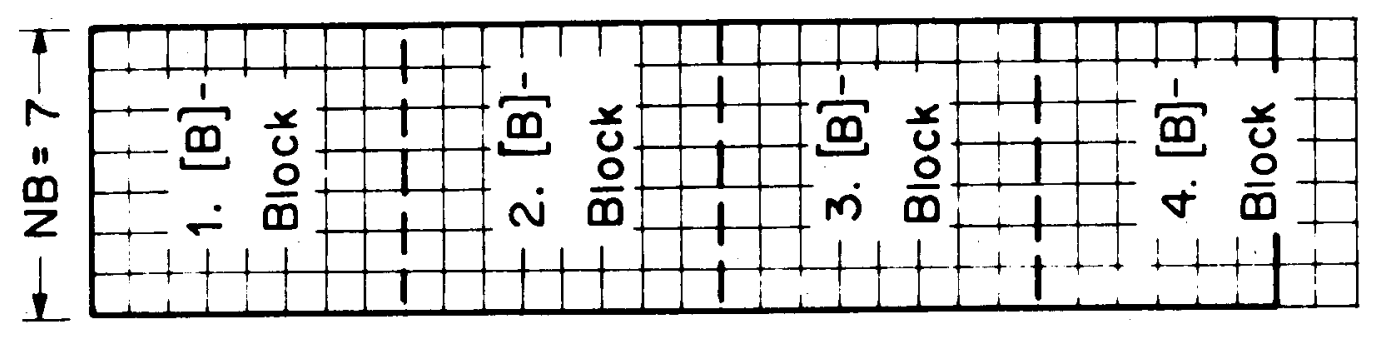

$+$

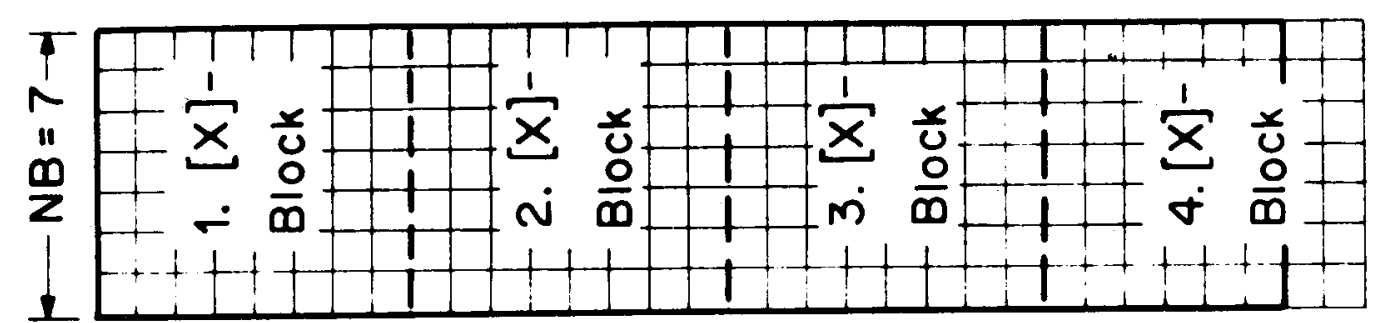

m

$\underline{\underline{\underline{\omega}}}$

号

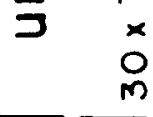

$x \underline{x}$

-

区完

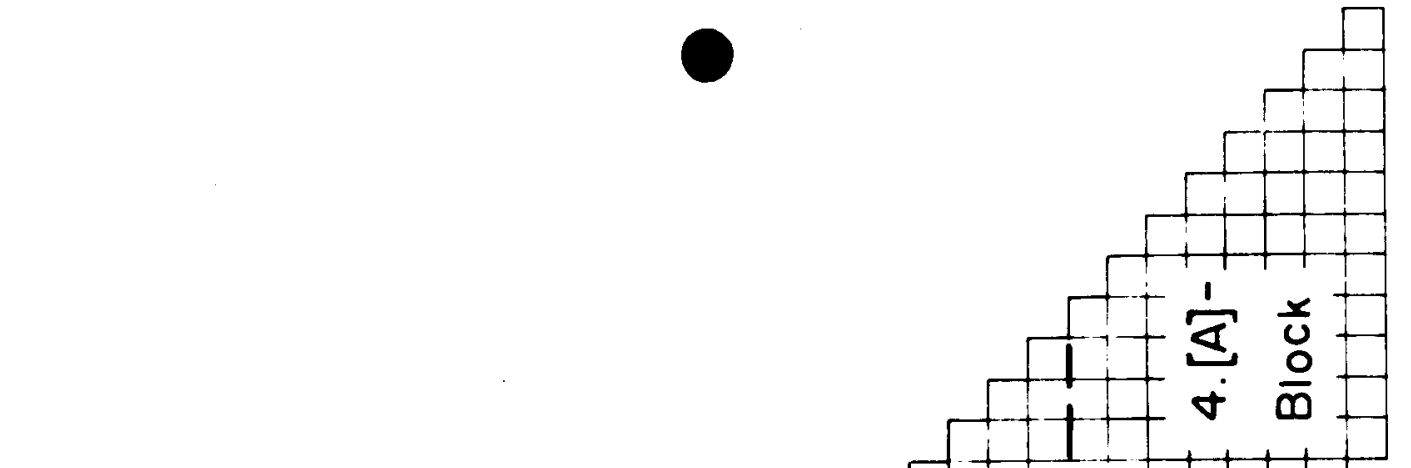

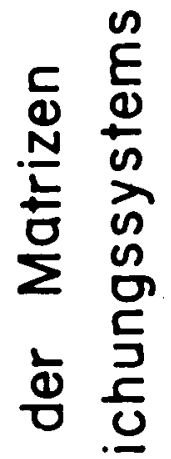

돈

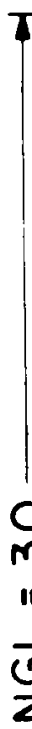

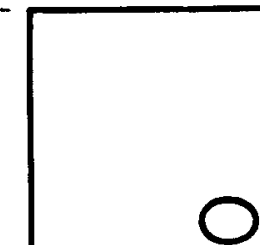

!

7

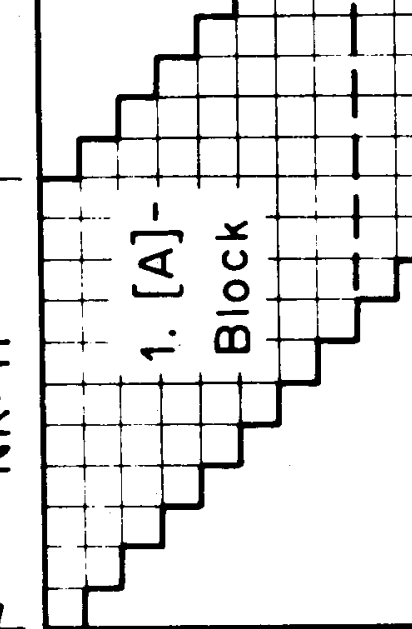

$\frac{1}{4} \div 11$

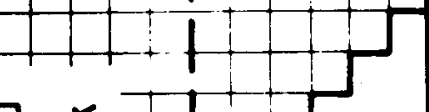

i $\frac{0}{m} 1$

2
$m$
$m$

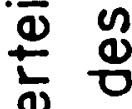

달

ㅁ

농

음

क

음

: $\frac{0}{0}$

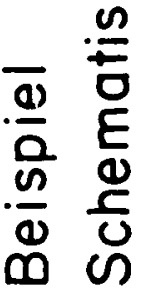

$\ddot{10}$

iv 


\section{$[\mathrm{X}]_{\mathrm{NGLXNB}} \cdot$}

Bei allen Matrizen werden die Koeffizenten zeilenweise gespeichert. Zum Beispiel werden die NKxNZ Koeffizenten eines [A]-Blockes wie folgt in sequentielle Adresse angeordnet

$$
a_{1,1} \quad a_{1,2} \cdots a_{1, N K} \quad a_{2,1} a_{2,2} \cdots a_{2, N K} \cdots a_{N Z, 1} a_{N Z, 2} \cdots a_{N Z, N K}
$$

Die Blockübertragung erfolgt immer via Grossraumspeicher mit Hilfe der Subroutinen READMS und WRITMS. Diese entsprechen den "FORTRAN-Extended Mass Storage" Subroutinen, welche in "FORTRAN-Extended Reference Manua1" (Control Data Pub. No. 60176600 Rev. A, Seite 5 - 10 und Appendix I-3) beschrieben sind. Die Subroutinen READMS und WRITMS werden durch folgende Anweisungen angerufen:

CALL READMS ( $\mathrm{n}, \mathrm{a}, 1, \mathrm{k})$

CALL WRITMS $(n, a, 1, k)$

Dabei bedeuten die formalen Parameter:

$\mathrm{n}$ : Identifikationsnummer der zu übertragenden Matrix oder auch logische Nummer des "Files", wo die entsprechenden Blöcke gespeichert sind $\left({ }^{*}\right)$.

a : Speicheradresse des ersten Wortes des Blockes.

1 : Länge des Blockes

k : Blocknummer.

Wird das Programm mit dem "FORTRAN-Extended Compiler" übersetzt, können die "Library"Subroutinen READMS und WRITMS verwendet werden. Ist das nicht der Fall, muss der Benützer diese Subroutinen selber schreiben.

\subsection{Die Subroutine BANDMAT}

Die symmetrische Koeffizentenmatrix $[A]_{\text {NGLXNGL }}$ kann als Produkt von drei Matrizen (Gauss'sche Zerlegung) betrachtet werden:

$$
[A]=[U][P][U]^{T}
$$

Dabei ist $[\mathrm{U}]_{\text {NGLXNGL }}$ eine untere Dreieckmatrix; deren Diagonalelemente aus "Einser" bestehen. $[\mathrm{P}]_{N G L x N G L}$ ist eine Diagonalmatrix, deren $\mathrm{p}_{\mathrm{kk}}$-Koeffizenten die Pivotelemente des Gauss'schen Algorithmus sind.

Die obere Dreiecksmatrix [D] ${ }_{\text {NGLXNGL }}$ (Halbinverse von $[A]$ ) ist wie folgt definiert:

$$
[D] \equiv[P][U]^{\mathrm{T}}
$$

Das Gleichungssystem schreibt sich dann:

$$
[A][X]+[B]=[U][P][U]^{T}[X]+[B]=[U][D][X]+[B]=0
$$

(*) Die n-Parameter für die Matrizen A, B, $X, D$ und $R$ werden von den formalen Parametern NTA, NTB, NTX, NTD und NTR gegeben. 
Die Subroutine BANDMAT ist im Stande, folgende Operationen durchzuführen:

1. Bildung der Matrix [D] aus [A] (Vorwärtselimination). Diese Operation wird durchgeführt, wenn der logische Parameter DBILD = .TRUE. ist. Falls die Identifikationsnummern der Matrizen [A] und [D] gleich sind (NTA = NTD), werden die Blöcke von [A] durch die Blöcke von [D] ersetzt. Das ist möglich, weil [D] die gleiche Bandstruktur besitzt wie [A]. Als Pivotelemente werden, der Reihe nach, die Diagonalelemente genommen. Lineare Abhängigkeiten in den Gleichungen werden nicht entdeckt.

2. Bildung der Lösungsmatrix $[X]$. Zwei Schritte sind dabei notwendig:

a. Bestimmung von $[U]^{-1}[B]$ (Vorwärtselimination). Es gilt:

$$
[D][X]+[U]^{-1}[B]=0
$$

Falls der logische Parameter DBILD = .FALSE. ist, wird $[U]^{-1}[B]$ aus der früher gerechneten halbinversen Matrix [D] bestimmt.

b. Bestimmung von $[X]$ (Rückwärtselimination). Es gilt:

$$
[X]=-[D]^{-1}[U]^{-1}[B]=-[A]^{-1}[B]
$$

Falls die Identifikationsnummern der Matrizen [B] und $[X]$ gleich sind (NTB = NTX), werden die Blöcke von $[B]$ durch die Blöcke von $[X]$ ersetzt.

Zwei [A]-Blöcke (jeder bestehend aus NZ·NK Wörtern) und zwei [B]-Blöcke (jeder bestehend aus NZ·NB Wörtern) müssen gleichzeitig im Hauptspeicher Platz finden. Die Anfangsadressen der entsprechenden Speicherzonen werden von den formalen Parametern A und B gegeben. Die Parameter NK (= halbe Bandweite von [A]) und NZ (= Anzahl Zeilen pro Block) können sonst beliebig gross sein. Wird NZ klein gewählt (NK hängt ja meistens von der Problemstellung ab), ist es möglich, mit wenig Speicherplatz sehr grosse G1eichungssysteme $z u$ behande1n. Ist jedoch $N Z \geq N K-1$, werden die Input - Output Kanäle weriger beansprucht, die Rechenzeit dürfte sich ebenfalls etwas verkürzen.

\section{$\underline{3.4}$ Die Subroutine BMRESID}

Die Subroutine BMRESID bestimmt aus der bekannten Lösungsmatrix [X] die Matrix der Residuenvektoren $[R]_{N G L x N B}$. Diese ist wie folgt definiert:

$$
[\mathrm{R}] \equiv[\mathrm{A}][\mathrm{X}]+[\mathrm{B}]
$$

Die Längen der Residuenvektoren werden ebenfalls bestimmt und dividiert durch die Längen der entsprechenden Belastungsvektoren. Diese Werte werden, beginnend in der Adresse <RLAENGE>, sequentiell gespeichert und können als Fehlermass benutzt werden.

Falls die Lösungsmatrix [X] mit numerischen Fehlern behaftet ist, ist es möglich, aus der Matrix der Residuenvektoren [R] eine verbesserte Lösungsmatrix [ $\left.X^{*}\right]$ zu bestimmen aus der Formel:

$$
\left[\mathrm{X}^{*}\right]=[\mathrm{X}]-[\mathrm{A}]^{-1}[\mathrm{R}]=[\mathrm{X}]+[\Delta \mathrm{X}]
$$

Die Bestimmung der Korrekturmatrix $[\Delta X]=-[A]^{-1}[R]$ ist besonders einfach, weil $[\Delta X]$ 
die Lösungsmatrix von folgendem Gleichungssystem ist:

$$
[A][\Delta X]+[R]=0
$$

Die Korrekturmatrix $[\Delta \mathrm{X}]$ kann folglich mit Hilfe der Subroutine BANDMAT bestimmt werden. Da die halbinverse Matrix [D] bei der ursprünglichen Lösung des Gleichungssystems schon bestimmt wurde, wird der dabei notwendige Rechenaufwand verhälnismässig klein sein.

Der Bedarf an Speicherplatz für die Subroutine BMRESID ist im Programm selbst mit Hilfe von "Comment"-Karten angegeben. 
ANHANG I : KONDENSATION DER INTERNEN FREIHEITSGRADE FUER NICHT GESTUETZTE UND FUER

ELASTISCH GESTUETZTE VIERECKIGE PLATTENELEMENTE

Behandelt wird zuerst der Fall von nicht gestutzten Plattenelementen.

Die Elemente VKPL28 und VKPL24 besitzen 10 "interne" Verschiebungsvariablen (4 Seitenvariablen und 6 Schwerpunktvariablen), die zusammen den Vektor $\left\{W_{i}\right\}$ bilden, sowie 28 bzw. 24 "externe" Variablen, die den Vektor $\{W\}=\left\{W_{e}\right\}$ bilden.

Die potentielle Energie $\Pi$ eines Plattenelementes, auf welchem eine Last $q(x, y)$ wirkt, ist :

$$
I=U-V=\frac{1}{2}\left\{\begin{array}{c}
w_{i} \\
\hdashline w_{e}
\end{array}\right\}\left[\begin{array}{c:c}
K_{i j} & k_{i e} \\
\hdashline K_{e i} & K_{e e}
\end{array}\right]\left[\begin{array}{c}
w_{i} \\
w_{e}
\end{array}\right\}-\left\{\begin{array}{c}
w_{i} \\
\hdashline w_{e}
\end{array}\right\}\left\{\begin{array}{c}
Q_{i} \\
Q_{e}
\end{array}\right\}
$$

Dabei werden die Steifigkeitsmatrix und der Lastvektor, dem Verschiebungsvektor entsprechend, in 4 Untermatrizen $\left[\mathrm{K}_{i i}\right],\left[\mathrm{K}_{i e}\right]=\left[\mathrm{K}_{e i}\right]^{\mathrm{T}}$ und $\left[\mathrm{K}_{e e}\right]$ bzw. in zwei Untervektoren $\left\{Q_{i}\right\}$ und $\left\{Q_{e}\right\}$ unterteilt.

Die potentielle Energie des aus vielen Elementen zusammengesetzten Systems ist am Lösungspunkt minimal. Da aber die internen Variablen des Vektors $\left\{W_{i}\right\}$ die nebenliegenden Elemente nicht beeinflussen, ist es möglich, das Minimumprinzip der potentiellen Energie im Innern des Elementes anzuwenden. Duch null-setzen der partiellen Ableitungen der potentiellen Energie nach allen internen Variablen bekommt man:

$$
\begin{aligned}
& \frac{\partial \pi}{\partial\left\{W_{i}\right\}}=\left[K_{i i}\right]\left\{w_{i}\right\}+\left[K_{i e}\right]\left\{w_{e}\right\}-\left\{Q_{i}\right\}=0 \\
& \text { oder } \\
& \left\{w_{i}\right\}=-\left[K_{i i}\right]^{-1}\left[K_{i i}\right]\left\{w_{e}\right\}+\left[K_{i i}\right]^{-1}\left\{Q_{i}\right\}
\end{aligned}
$$

Durch Einsetzen dieser Beziehungen in den Ausdruck für II gelingt es, den Vektor $\left\{W_{i}\right\}$ zu eliminieren:

$$
\begin{aligned}
& \Pi=\frac{1}{2}\left\{W_{e}\right\}^{T}\left(\left[K_{e e}\right]-\left[K_{e i}\right]\left[K_{i i}\right]^{-1}\left[K_{i e}\right]\right\}\left\{W_{e}\right\}-\left\{W_{e}\right\}^{T}\left(\left\{Q_{e}\right\}-\left[K_{e i}\right]\left[K_{i i}\right]^{-1}\left\{Q_{i}\right\}\right) \\
& \Pi=\frac{1}{2}\left\{W_{e}\right\}^{T}[K]\left\{W_{e}\right\}-\left\{W_{e}\right\}^{T}\{Q\}
\end{aligned}
$$

wobei:

$$
[K] \equiv\left[K_{e e}\right]-\left[K_{e i}\right]\left[K_{i i}\right]^{-1}\left[K_{i e}\right]
$$

die gesuchte "kondensierte" Steifigkeitsmatrix und:

$$
\{Q\}=\left\{Q_{e}\right\}-\left[K_{e i}\right]\left[K_{i i}\right]^{-1}\left\{Q_{i}\right\}
$$


der "kondensierte" Lastvektor ist.

Der Algorithmus, welcher zur numerischen Bestimmung von [K] und $[Q]$ führt, ist ăusserst einfach. Für 10 interne Variablen genügen nämlich 10 Gauss'sche Eliminationsschritte, ausgefuhrt an folgender zusammengesetzter Matrix:

$$
\left[\begin{array}{c:c:c}
K_{i i} & K_{i e} & Q_{i} \\
\hdashline K_{e i} & K_{e e} & Q_{e}
\end{array}\right]
$$

um $[K]$ und $\{Q\}$ in der unteren Hălfte des entsprechenden Tableaus $z u$ erhalten. Dabei sind als Pivotelemente die Diagonalelemente der Matrix $\left[K_{i i}\right]$ zu nehmen. Man erhält:

$$
\left[\begin{array}{c:c:c}
D & -U^{-1} K_{i} & -U^{-1} Q_{i} \\
\hdashline K_{e e}-K_{e i} K_{i i}^{-1} K_{i e} & Q_{e}-K_{e i} K_{i i}^{-1} Q_{i}
\end{array}\right]
$$

Wobei [D] und [U] die obere und untere Dreiecksmatrizen der Gauss'schen Zerlegung von $\left[K_{i i}\right]$ sind $\left(\left[K_{i i}\right]=[U][D]\right)$.

Zur Behandlung von elastisch gestütten viereckigen Plattenelementen wird der gleichmässig verteilte Stützendruck $s$ einfach als eine zusätzliche interne Variable betrachtet. Dabei muss, an Stelle der potentiellen Energie $\pi$, eine neue Funktion $\pi^{*}$ verwendet werden, welche den Einfluss des Stützendruckes berücksichtigt:

$$
\pi^{*} \equiv \pi-\frac{1}{2} C \cdot F^{2} \cdot s^{2}+s \cdot \iint w d F
$$

Die Formänderungsarbeit der Stütze wird vom zweiten Summand gegeben. Der dritte Summand stellt die Arbeit des Stützendruckes s für ein Plattendurchbiegung $w$ dar $\left(^{*}\right)$.

Aus der Definition des Belastungsvektors $\{P\}$ für eine gleichmässig verteilte Belastung $p=1$ (s. Abschnitt 2.3) folgt:

$$
\iint w d F=\{W\}^{T}\{P\}=\left\{\begin{array}{c}
w_{i} \\
\hdashline W_{e}
\end{array}\right\}^{T}\left\{\begin{array}{c}
P_{i} \\
-P_{e} \\
P_{e}
\end{array}\right\}
$$

Für $\Pi^{*}$ kann man dann schreiben:

$$
\Pi^{*}=\pi-\frac{1}{2} \cdot C \cdot F^{2} \cdot s^{2}+s \cdot\left\{\begin{array}{c}
W_{i} \\
-W_{e}
\end{array}\right\}^{T}\left[\begin{array}{l}
P_{i} \\
-P_{e}
\end{array}\right\}
$$

oder:

(*) Durch null-setzen der partiellen Ableitung von $\pi^{*}$ nach $s$ bekommt man denselben Ausdruck wie in Abschnitt 2.6:

$$
\frac{\partial \pi}{\partial s}=-C \cdot F^{2} \cdot s+\iint w d F=0 \text { oder } \frac{1}{F} \iint w d F=C \cdot F \cdot s
$$




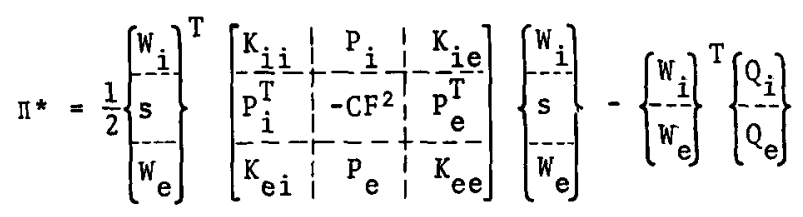

Die internen Verschiebungsvariablen des Vektors $\left\{W_{i}\right\}$ sowie die Stützendruckvariable $s$ werden, wie bei den nicht gestützen Elementen, eliminiert, wobei für s einfach ein zusätzlicher Eliminationsschritt notwendig ist. Man erhält damit die "kondensierte" Steifigkeitsmatrix:

$[K] \equiv\left[K_{e e}\right]-\left[K_{e i}\right]\left[K_{i j}\right]^{-1}\left[K_{i e}\right]+\frac{\left(\left\{P_{e}\right\}-\left[K_{e i}\right]\left[K_{i i}\right]^{-1}\left[P_{i}\right\}\right)\left(\left\{P_{e}\right\}-\left[K_{e i}\right]\left[K_{i i}\right]^{-1}\left\{P_{i}\right\}\right)^{T}}{C \cdot F^{2}+\left\{P_{i}\right\}^{T}\left[K_{i j}\right]^{-1}\left\{P_{i}\right\}}$

und den "kondensierten" Belastungsvektor:

$$
\{Q\} \equiv\left\{Q_{e}\right\}-\left[K_{e i}\right]\left[K_{i i}\right]^{-1}\left[K_{i e}\right]+\frac{\left(\left\{P_{e}\right\}-\left[K_{e i}\right]\left[K_{i j}\right]^{-1}\left\{P_{i}\right\}\right)\left\{P_{i}\right]^{T}\left[K_{i j}\right]^{-1}\left(Q_{i}\right\}}{C \cdot F^{2}+\left[P_{i}\right\}^{T}\left[K_{i i}\right]^{-1}\left\{P_{i}\right\}}
$$

Falls auf dem Plattenelement eine gleichmässig verteilte Last $\mathrm{p}$ wirkt (so dass die Lastverteilung und die Stützendruckverteilung gleich sind), bekommt man für den "kondensierten" Belastungsvektor $\{P\}$ den etwas einfacheren Ausdruck:

$$
\{P\}=\left(\left\{P_{e}\right\}-\left[K_{e i}\right]\left[K_{i \mathbf{i}}\right]^{-1}\left\{P_{i}\right\}\right)\left(1-\frac{1}{1+C \cdot F^{2} /\left\{P_{i}\right\}^{T}\left[K_{i \mathbf{i}}\right]^{-1}\left\{P_{\mathbf{i}}\right\}}\right)
$$

Ist die Stützenkonstante $C$ unendlich gross (für nicht gestützte Elemente), verschwindet der Einfluss von $s$. Ist $C=0$ (für frei drehbare jedoch unsenkbare Elemente, bei denen $\iint \mathrm{w} d F=0$ sein muss), verschwindet der Einfluss einer gleichmässig verteilten Last $\mathrm{p}$.

Es sei noch erwähnt, dass diese Prozedur für Elemente, die keine interne Verschiebungsvariablen besitzen (z.B. die Dreieckelemente DRPL21 undDRPL18), ungeeignet ist. Wenn $\mathrm{C}=0$ ist, wird es nämlich unmöglich sein, die interne Variable $s \mathrm{zu}$ eliminieren, weil das entsprechende Pivotelement Null wird. Erst bei der Lösung des globalen Gleichungssystems könnte die Variable s eliminiert werden.

Fig. 6 zeigt einige numerische Resultate für eine quadratische doppelsymmetrische P1atte, die in der Mitte auf einer quadratischen Stütze liegt. Zwei Ränder der Platte sind eingespannt und zwei sind frei drehbar gelagert. Die Platte ist isotropisch und hat folgende. Steifigkeitskoeffizenten: $d_{11}=d_{22}=1,0 ; d_{12}=0,2 ; d_{33}=0,8(v=0,2)$ Damit die Resultate mit dem PLATE-FOURIER Programm (s. Lit. ${ }^{8}$ ) direkt vergleichbar sind, ist die Stützenkonstante $C$ so gewählt $(C=4,0372522)$, dass die Plattendurchbiegung in der Mitte der Stütze Null wird. Die Elementeinteilung (VKPL28 - Elemente) ist in dünnen Linien gezeichnet. Es ergibt sich damit für die 512 unbekannten Verschiebungsvariablen eine Koeffizentenmatrix mit einer halben Bandweite $N K=78$. Die Lösung dieses Gleichungssystems mit der Subroutine BANDMAT verlangt ca. 45 Sek, auf einem CDC 6500-Computer. 


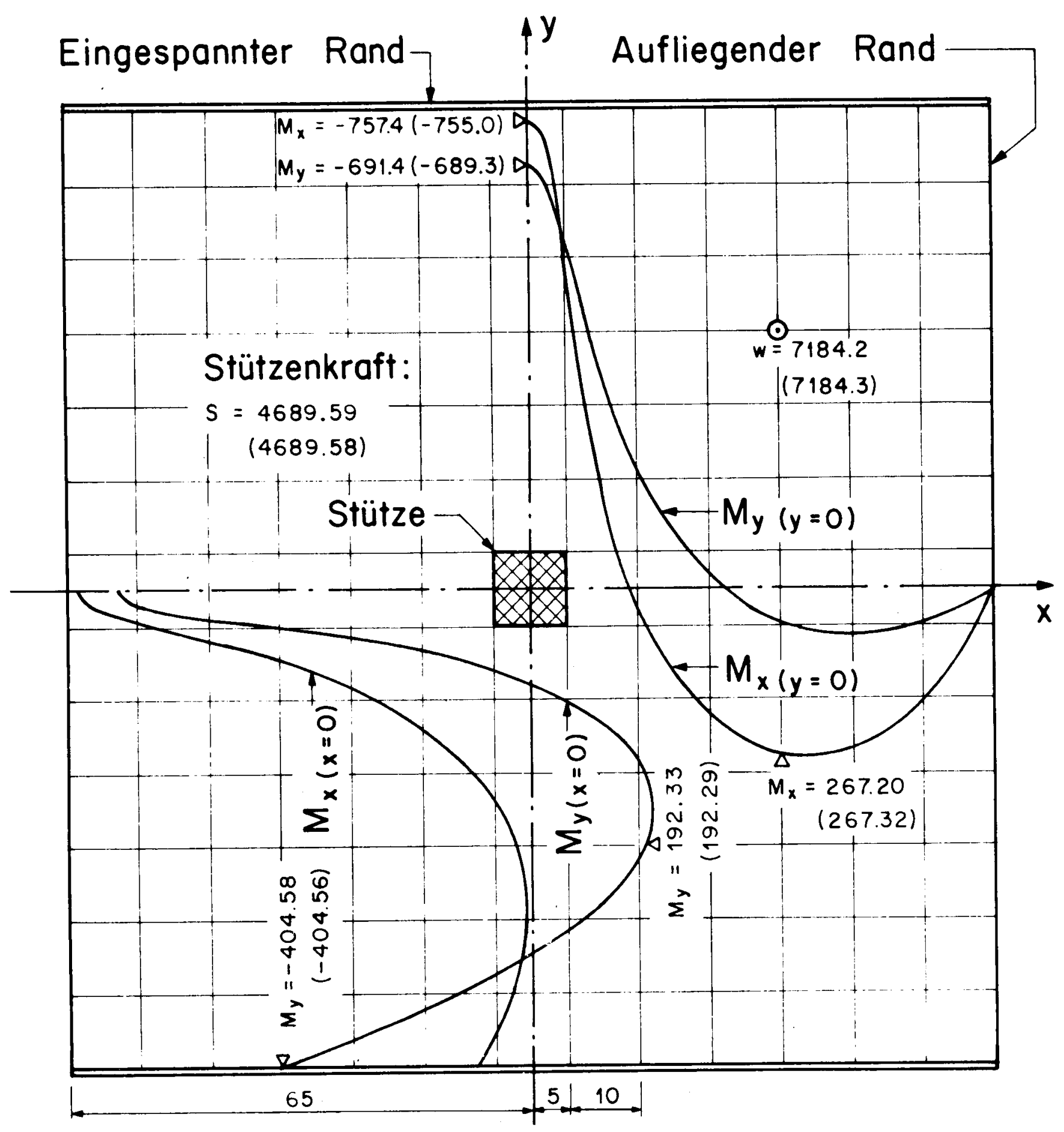

Fig. 6: Numerische Resultate bei einer gleichmässig belasteten quadratischen Platte mit einer Stütze in der Mitte. 
Der Verlauf der Biegemomente $M_{x}$ und $M_{y}$ entlang den Symmetrieebenen für eine gleichmässig verteilte Belastung $p=1$ ist in Fig. 6 gezeigt. Der Wert des Stützendruckes sowie einige diskrete Werte der Momente und der Durchbiegungen sind ebenfalls angegeben und mit den entsprechenden Werten aus dem PLATE-FOURIER Programm (in Klammer gezeigt) verglichen. 
1. Bosshard W.: "Ein neues, vollverträgliches endliches Element für Plattenbiegung", Internationaler Verein für Brückenbau und Hochbau, Abhandlungen, Band 28-I, 1968.

2. Goë1 J.J.: "Utilisation numerique de la methode de Ritz. Application aux plaques" Thèse présentêe à 1'Ecole Politechnique de 1'Universitê de Lausanne, 1967.

3. Ball K.: "A Refined Triangular Plate Bending Finite Element", Int. Journal for Numerical Methods in Engineering. Vol. 1, No. 1, 1969.

4. Argyris J.H./Fried I./Scharpf D.W.: The TUBA Family of Plate Elements for the Matrix Displacement Method", The Aeronautical Journal of the Royal Aeronautical Society, Vo1. 72, August 1968.

5. Felippa C.A.: "Refined Finite Element Analysis of Linear and Nonlinear Two-Dimensional Structures", SESM Report No. 66-22, University of California, Berkeley, California, 1966.

6. Carr A.J.: "A Refined Element Analysis of Thin Shell Structures Including Dynamic Loadings", SESM Report No. 66-22, University of California, Berkeley, California, Juni 1967.

7. Bergan P.G.: "Plane Stress Analysis using the Finite Element Method. Triangular Element with 6 Parameters in each Node", Division of Structural Mechanics, The Technical University of Norway, Trondheim, 1967.

8. Pfaffinger D./Thürlimann B.: "Tabellen für unterzugslose Decken", Verlags-AG der akademischen technischen Vereine Zürich, 1967. 


\section{TEIL II : PROGRAMME}

\section{SUBROUTINE DRPL21}

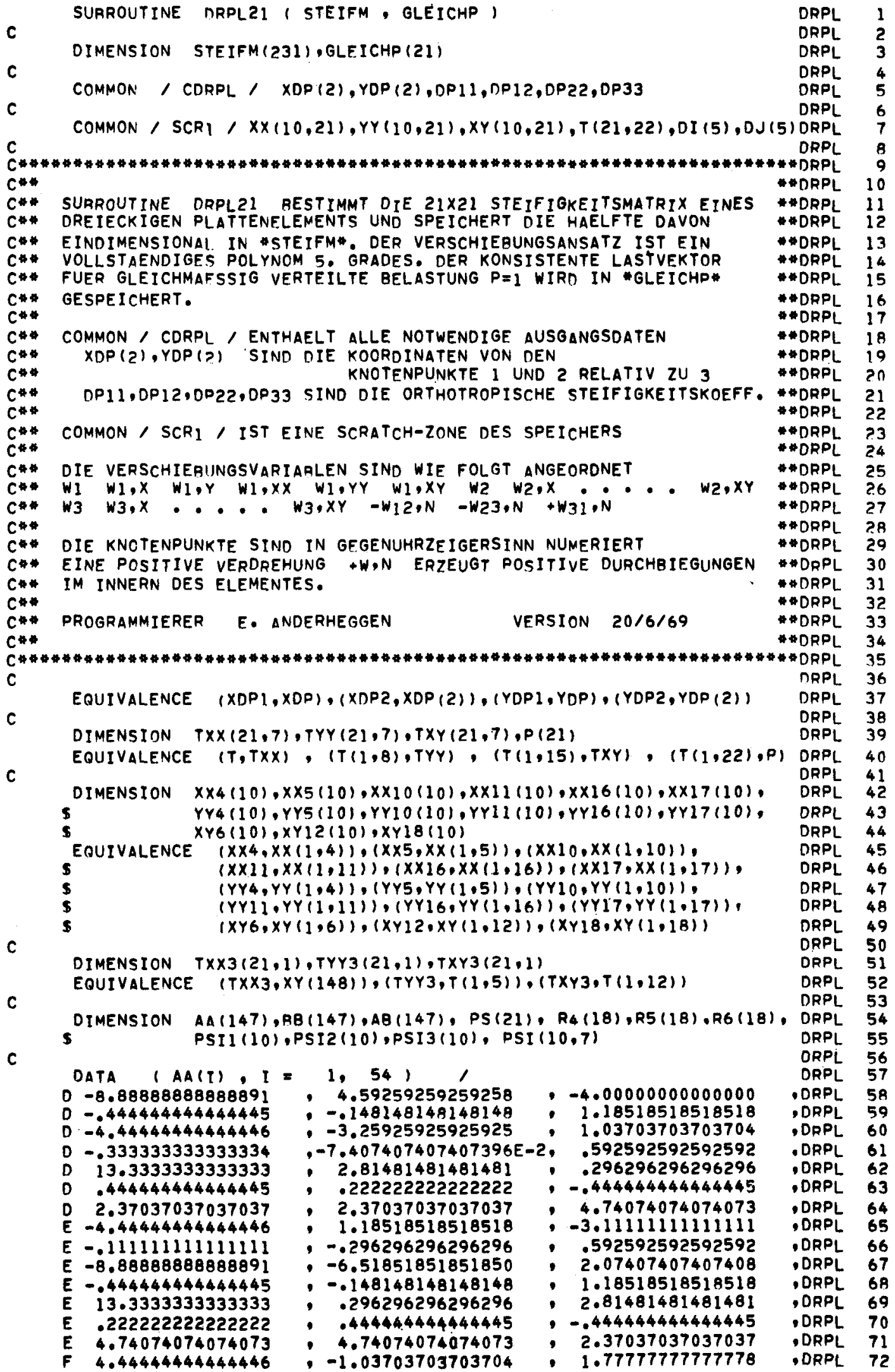




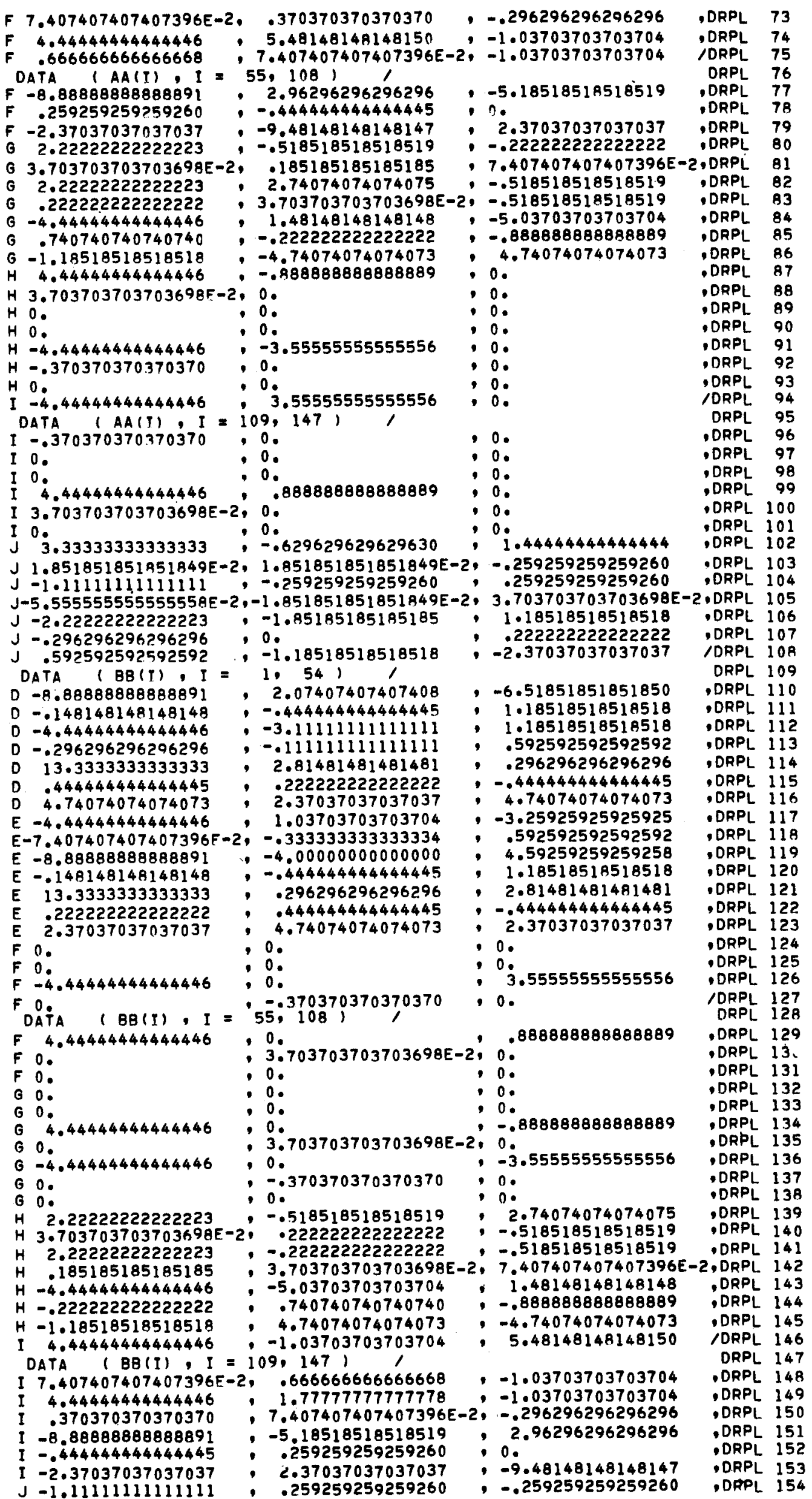




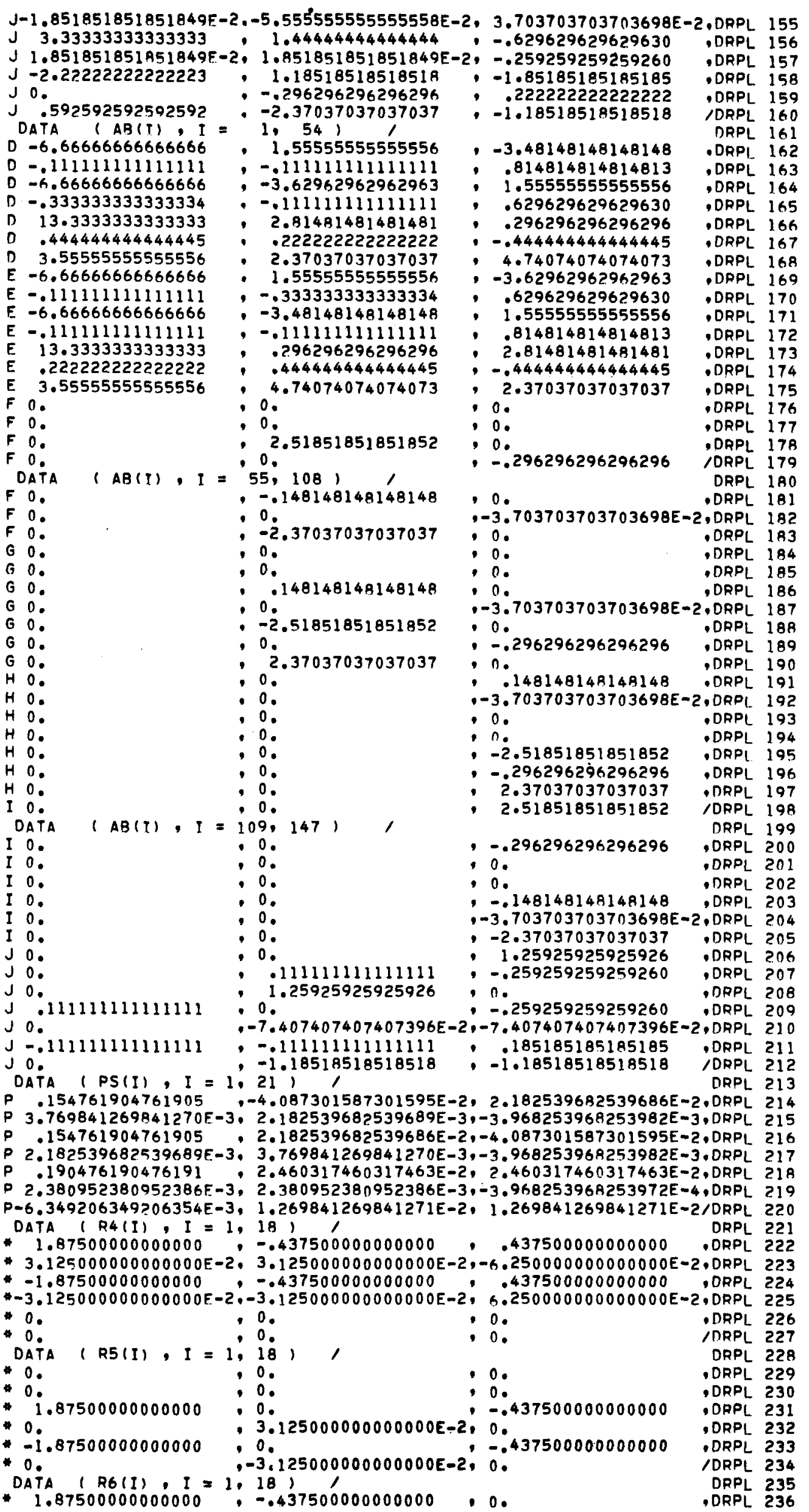




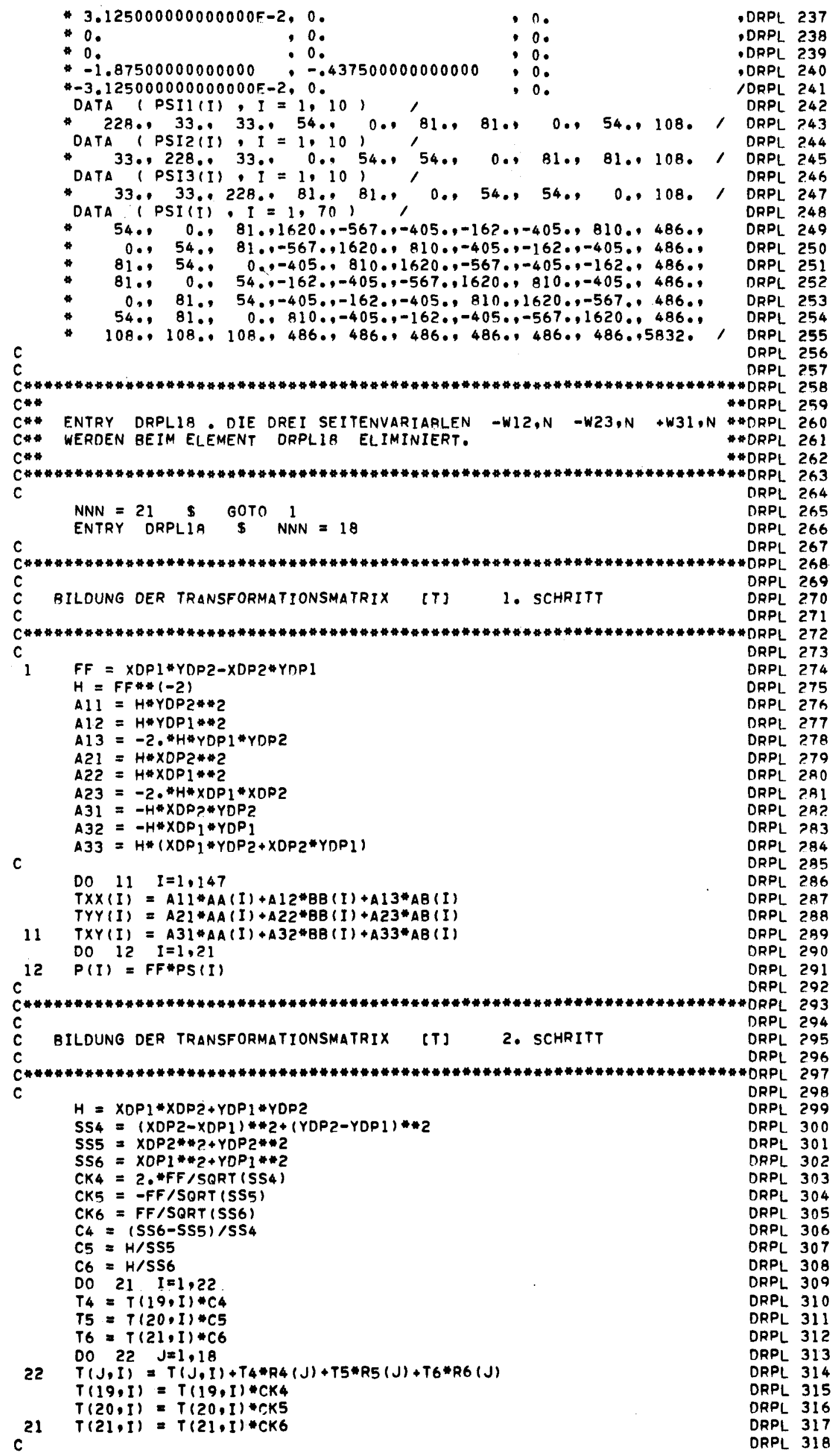




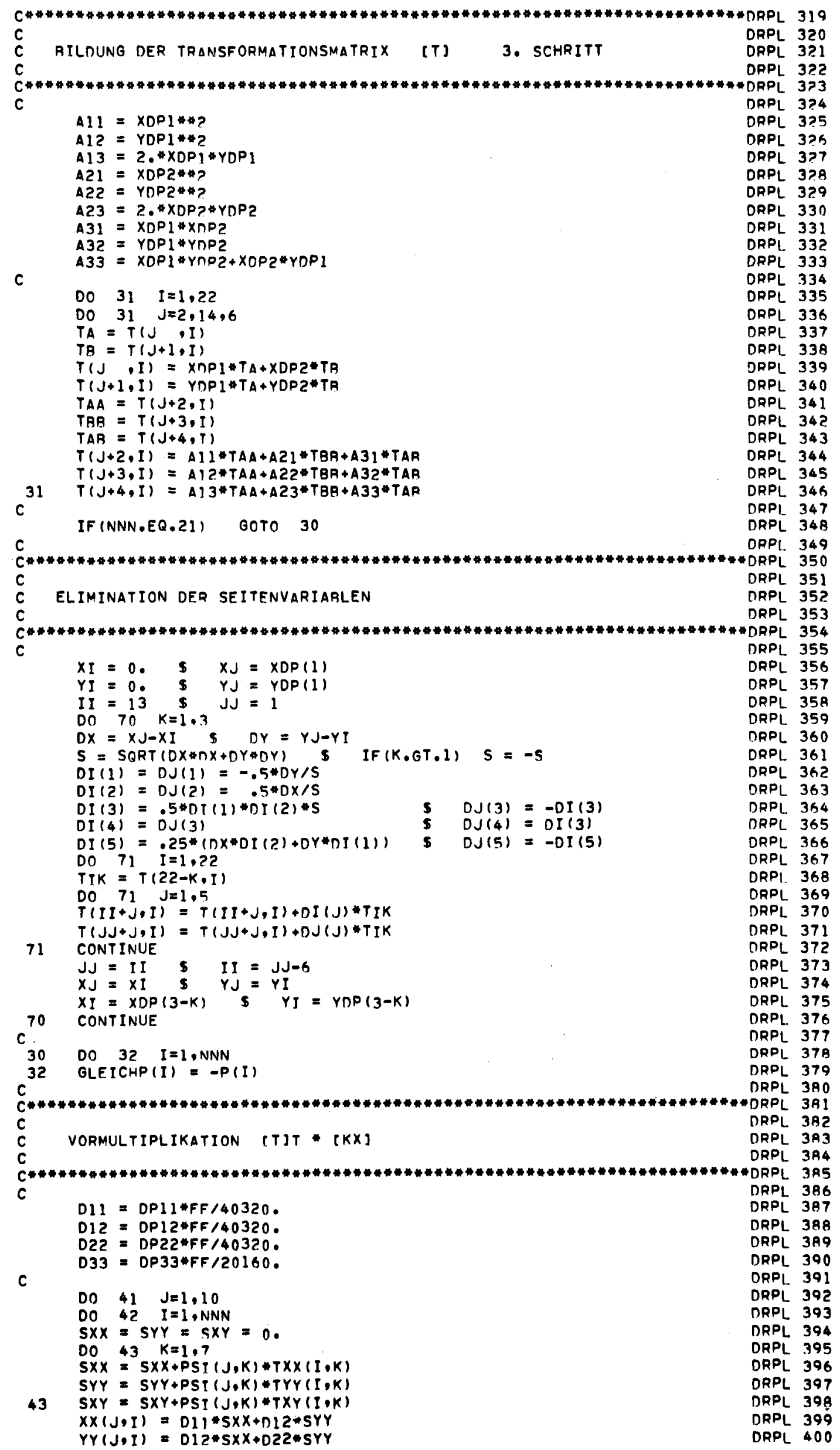


$42 X Y(J, I)=D 33 * 5 X Y$

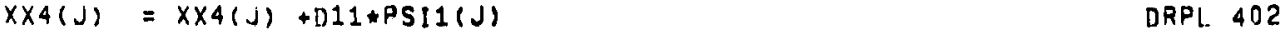

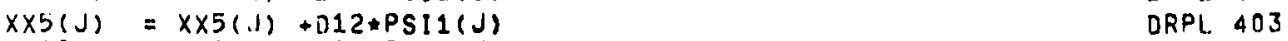

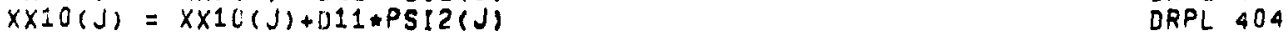

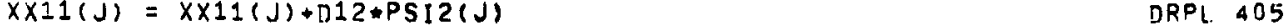

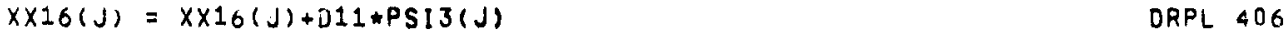

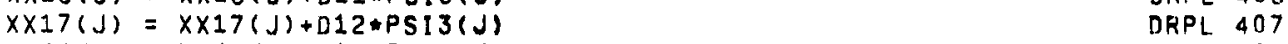

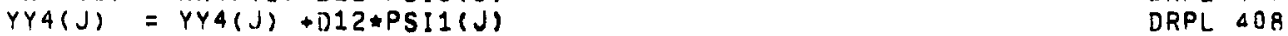

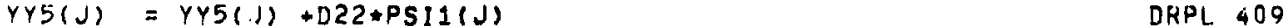

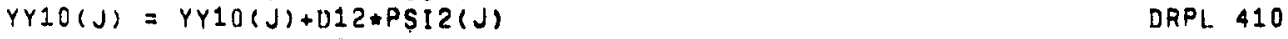

$Y Y 11(J)=Y Y 11(J)+D 22 * P S I 2(J) \quad$ DRPL 411

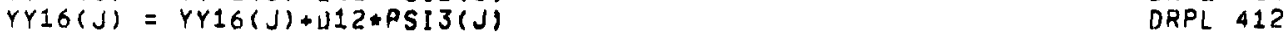

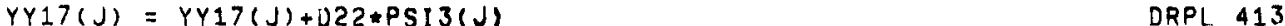

$X Y G(J)=X Y G(J)+D 33 * P S I 1(J) \quad$ DRPL 414

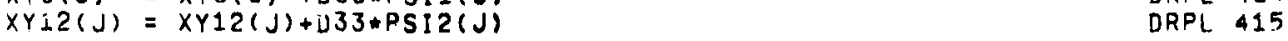

$41 X Y 18(\mathrm{~J})=X Y 18(\mathrm{~J})+D 33 * P S I 3(\mathrm{~J}) \quad 416$

C

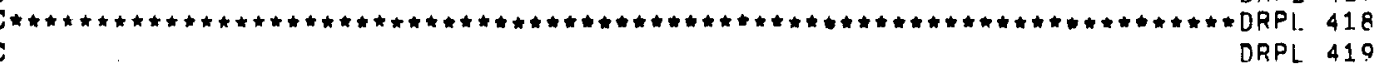

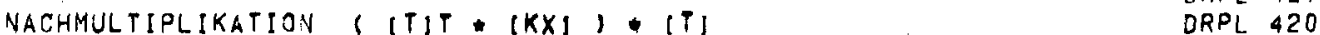

C

C

$\begin{array}{ll}I J=1 & \text { DRPL } 424\end{array}$

$\begin{array}{ll}0051 \mathrm{~J}=1, N \mathrm{~N} N & \text { DRPL. } 425\end{array}$

DO $51 I=J, N N N \quad$ DRPL 426

DRPL 427

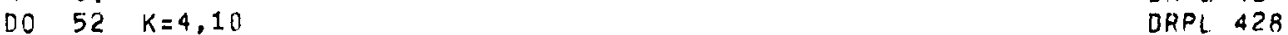

52. $H=H+X X(K, I)-T X X 3(J, K)+Y Y(K, I) * T Y Y 3(J, K)+X Y(K, 1) * T X Y 3(J, K) \quad$ DRPL 429 STEIFM(IJ)=H DRPL 430

$511 J=I J+1 \quad$ DRPL 431

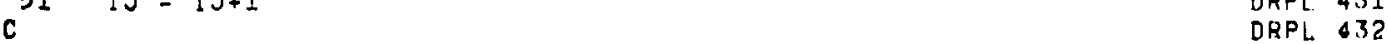

IJ $=3 * U N N-2 \quad$ DRPL 433

DO $53 . K=1,3 \quad$ DRPL 434

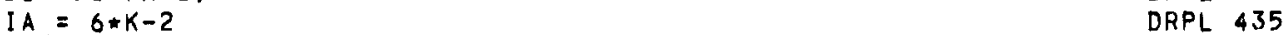

DO $54 \quad I=1 \mathrm{~A}$, iViNY DRPL 436

STEIFM(IJ)=STEIFM(IJ)+XX(K,I) $\quad$ DRPL 437

54 IJ $=I J+1 \quad$ DRPL 438

$I A=I A+1 \quad$ DRPL 439

DO $55 \mathrm{I}=1 \mathrm{~A}, N N \mathrm{DN} \quad$ DRPL 440

STEIFM(IJ)=STEIFIM IJ)+YY(K,I) $\quad$ DRPL 441

$I A=I A+1 \quad$ DRPL 443

DO $56 I=I A, N N N \quad$ DRPL 444

STEIFM(IJ) $=\operatorname{STEIFI(IJ)+XY(K,I)} \quad$ DRPL 445

$I J=I J+1 \quad$ DRPL $\cdot 446$

$\begin{array}{lll}53 & I J=I J+60-18 * K-(21-i N N N)+3 & \text { DRPL } 447\end{array}$

RETURN

DRPL 448

DRPL 449

DRPL 450

END

DRPL 451

\section{SUBROUTINE DRSC18}

SUBROUTINE DRSCI8 (STEIFM, OLEICHU, GLEICHV)

C

DIMENSION STEIFH(171), GLEICHU(18), OLEICHV(18)

COMMON, CDRSC, XDS(2),YDS(2),DS12,DS12,DS22,DS33

COMMON / SCR1, TS(20,20),T $(20,20)$

DRSC

DRSC 2

$\begin{array}{ll}\text { DRSC } & 2 \\ \text { DRSC } & 3\end{array}$

DRSC 4

DRSC 6

DRSC 7

DRSC 6

C

C**
\#DRSC,

C*: SUBROUTINE 'DRSC18, BESTIMMT DIE $18 \times 18$ STEIFIGKEITSMATRIX

$C *$ EINES DREIECKIGE: SCHEIBEELEMENTS, DIE HAELFTE DAVON WIRD * *RSC

C** EINDIMENSIONAL IN \#STEIFM* OESPEICHERT. DIE VERSCHIERUNGS - \#DRSC

C* ANSAETZE IN U- UNO V-RICHTUNG SIND VOLLSTAENDIGE POLYNOME $\quad$ औDRSC

C** 3. GRADES. IN \#GLEICHU* UND *GLEICHV* WERDEN DIE KONSISTENTE *DRSC.

C* LASTVEKTOREN FUER GLEICHMAESSIGE GELASTUNOEN $P=1$ IN U* UND V- **DRSC

C** RICHTUNG GESPEICHERT.

$c \star *$

C** COMMON / CURSC / ENTHAELT ALLE NOTWENDIGE AUSGANGSDATEN

$\begin{array}{ll}\rightarrow \text { DRSC } & 17 \\ \rightarrow \text { DRSC } & 18\end{array}$

$\begin{array}{ll}\rightarrow \text { DRSC } & 18 \\ -\rightarrow \text { DRSC } & 19\end{array}$

* DRSC 20

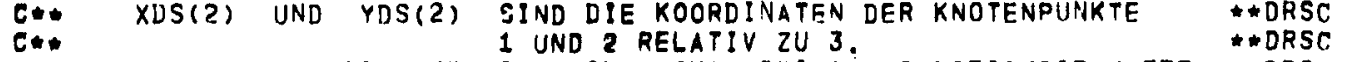

C* DS11,DS12,DS?2,DS33 SIND DIE ORTHOTROPISCHE STEIFIGKEITSKOEFF, \#DRSC 


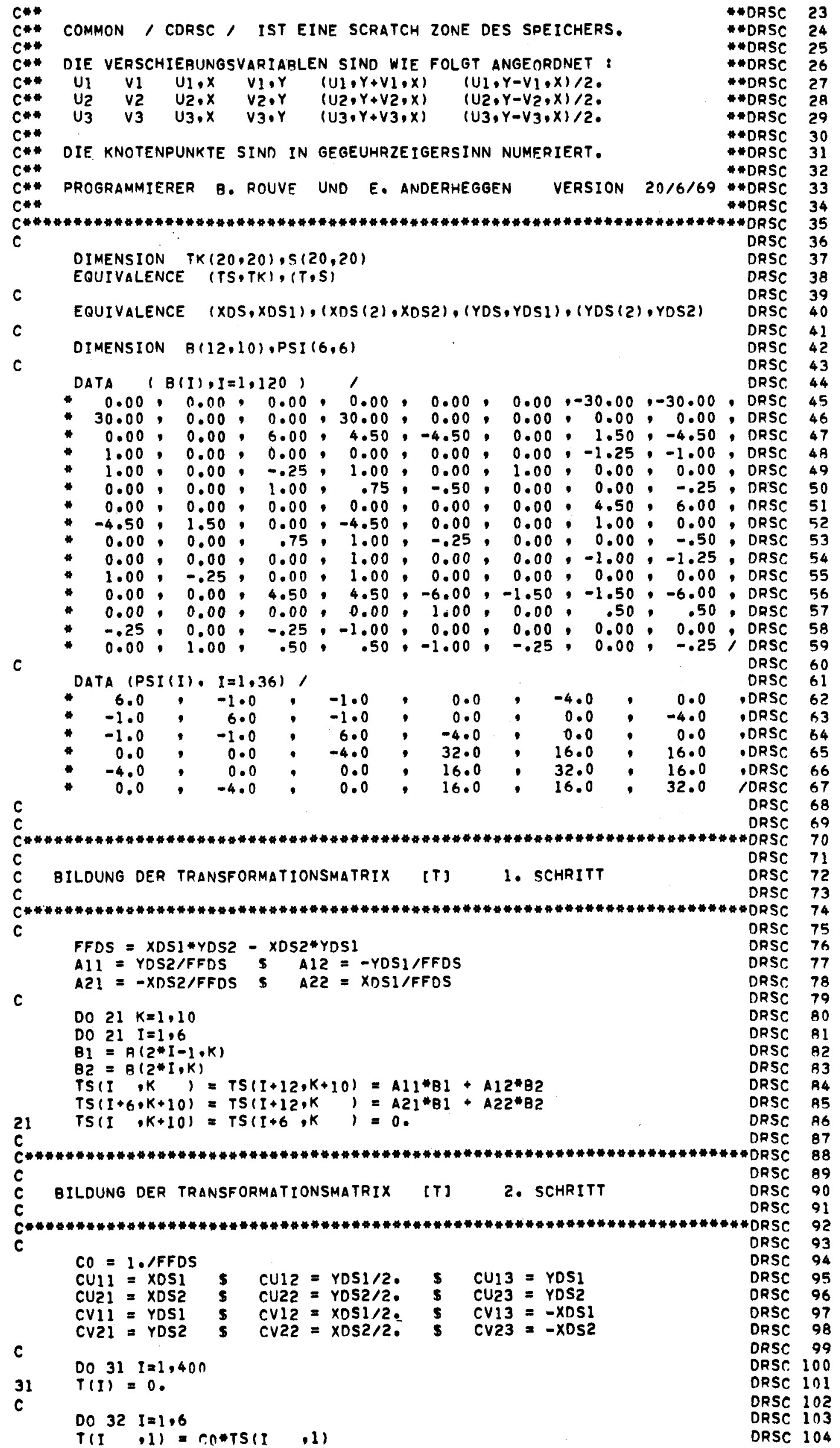


$T(I+12,1)=\operatorname{CO} * T S(I+12,1)$

DRSC 105

$T(I+6,2)=\operatorname{CO} T S(T+6,11)$

DRSC 106

$T(I+12,2)=\cot T(I+12,11)$

DRSC 107

DRSC 108

DRSC 109

DRSC 110

DO $32 K=3,15,6$

$T(T, K)=T S(I, K I)$,

DRSC 111

DRSC 112

DRSC 113

DRSC 114

$T(I+6, K+1)=T S(I+6, K I+10)$

DRSC 115

C

$T(I+12, K+1)=T S(T+12, K 1+10)$

DRSC 116

DRSC 117

DRSC 118

DRSC 119

DRSC 120

DRSC 121

DRSC 122

DRSC 123

DRSC 124

DRSC 125

DRSC 126

DRSC 127

DRSC 128

DRSC 129

DRSC 130

DRSC 131

C

$\begin{aligned} T(I+12, K+3)= & \text { CVII TS }(I+12, K 2+10)+\text { CV } 1 * T S(I+12, K 2+11) \\ T(I+12, K+4)= & \text { CUI2*TS }(I+12, K 2,+ \text { CU22*TS }(I+12, K 2+1) \\ & \text { CVI2*TS }(I+12, K 2+10)+\text { CV } 22 * T S(I+12, K 2+11)\end{aligned}$

DRSC 132

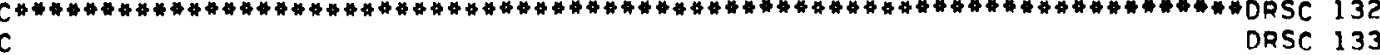

$\begin{array}{lll}C & \text { VORMULTIPLIKATION }[T K]=[T] *[K S] & \text { DRSC } 133 \\ C & \text { DRSC } 134\end{array}$

DRSC 135

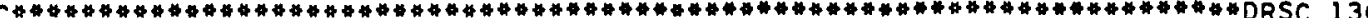

$D 11=D S 11 * F F D S / 360$.

$D 22=D S 224 F F D S 1360$

DRSC 137

DRSC 138

DRSC 139

$D 12=D S 12 \$ F F \cap 51360$

DRSC 140

D33 $=$ DS33 FFOS/360.

DRSC 141

DRSC 142

DRSC 143

$0041 \mathrm{~K}=1,6$

DO $41 \quad I=1,20$

$H_{1}=H_{2}=H_{3}=0$.

DO $42 \mathrm{~J}=1,6$

$H_{1}=H 1+T(1$, I $) \# P S I(J, K)$

DRSC 144

DRSC 145

DRSC 146

DRSC 147

$H 2=H 2+T(J+6, I) * P S I(J, K)$

DRSC 148

DRSC 149

DRSC 150

DRSC 151

DRSC 152

DRSC 153

DRSC 154

$T K(I, K+6)=012 * H 1$
$T K(I, K+12)=033 * H 3$

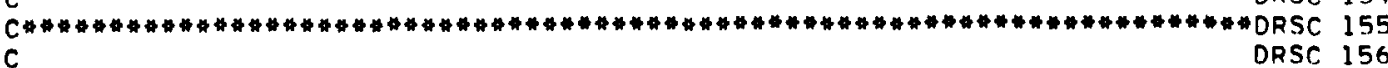

$\begin{array}{lll}C & \text { NACHMULTIPLIKATION [K] }=[K S] * \text { [T]T } & \text { DRSC } 156 \\ C & \text { DRSC } 157\end{array}$

DRSC 158

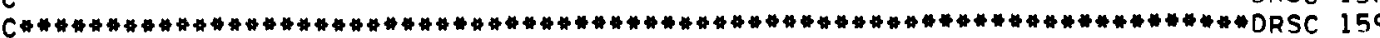

DO $60 \quad I=1,20$

DO $60 \mathrm{~J}=\mathrm{I} \cdot 20$

$H=0$.

DO $6 i \quad K=1, ? 0$

$61 \quad H=H+T K(I, K) * T(K, J)$

$60 \quad S(J, I)=H$

C

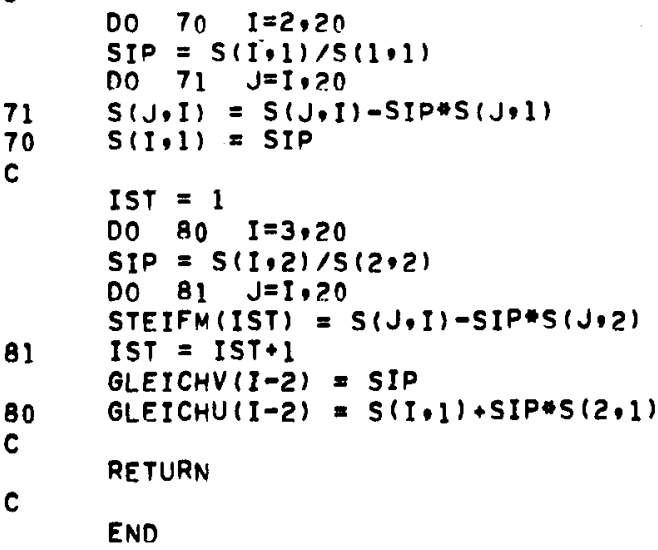

ORSC 160 DRSC ISI

DRSC 162

DRSC 163

DRSC 164

DRSC 165

DRSC 166

DRSC 167

DRSC 168

DRSC 169

DRSC 170

DRSC 171

DRSC 1.72

DRSC 173

DRSC 174

ORSC 175

DRSC 176

DRSC 177

DRSC 178

DRSC 170

DRSC 180

DRSC 181

DRSC 182

DRSC 183

DRSC 184

DRSC 185

DRSC 186 


\section{SUBROUTINE VKPL28}

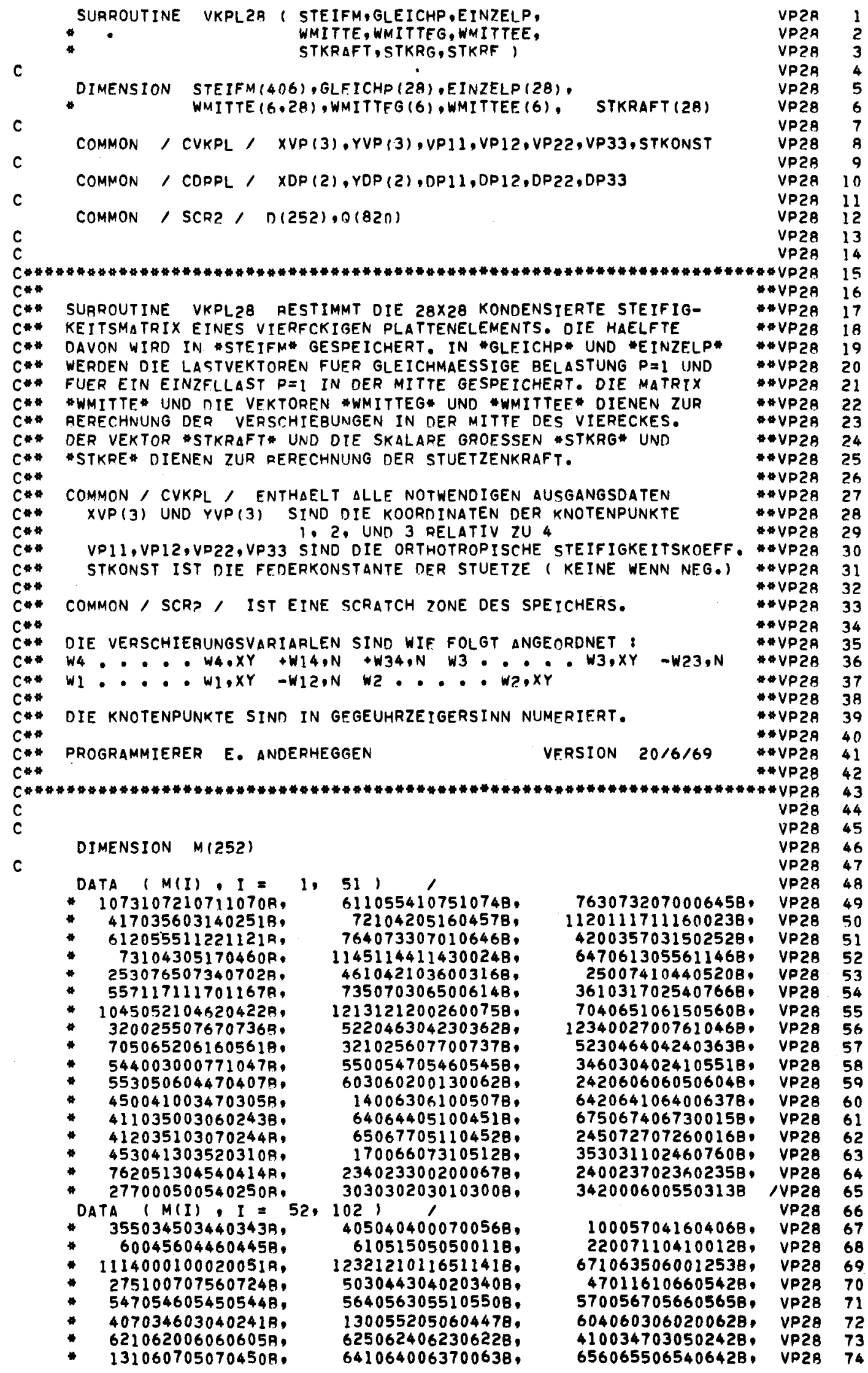


- 243066106600657A, 7070675067406738 , 6760511045204128 . $310024507460745 B$, $775077407730772 B$,

- 12750067013507618 . $1022052504660426 \mathrm{~A}$, $467042703660324 \mathrm{~A}$, $430036703250262 A$,

DATA I $M(1), I=103$

- 431037003260263E. 4720432037103278 . $1027053204730433 B$. $277005401220247 R$, $354034503440343 B$, 1260455044604458 , 6000051005201208 , $275140613741361 \mathrm{~B}$. 13001277127612758 . $426036503230260 R$, - $1323132213201317 \mathrm{~A}$. $215132105260467 \mathrm{~A}$, 2621344134313428 . $262134413431342 R^{\circ}$
$-1353135113501347 R^{\circ}$ - 13520530047104318 . - 3270264137313728 . - 14031402140114008 . DATA ( M(I) $I=154$

14110534047504358. $476043603750333 \mathrm{R}$. 437037603340271 A, 4400377033502729 , $501044104000336 R$, $1416054105020442 R$. $277012201670266 A_{0}$ $373034503440343 A_{\text {, }}$ $173047404460445 R$, $1312012001210166 \mathrm{R}$ $275146014551451 R$ $1424142314221421 R$, $435037403320267 A^{2}$, $1134110714351434 \mathrm{R}$ $42.126605350476 \mathrm{~A}$ $271124712 ? 61204 \mathrm{~A}$

DATA ( MII) I I = 205,

- $1111145014471446 R$,

$1270053705000440 \mathrm{R}$. 3360273125112308 . $1207116411401113 R$

10700230004412728 . $1076051604570417 R$. $460042003570315 R$, $421036003160253 \mathrm{R}$. $422036103170254 \mathrm{R}$, $463042303620320 \mathrm{~B}$ $1235052304640424 \mathrm{R}$. $277016700050257 \mathrm{~A}$, $364034503440343 A^{\circ}$ $11046504460445 R$.

- 14270166000400018. $275125312321210 \mathrm{~B}$,

C c C c*

BILDUNG DER NICHT KONDENSIERTEN STEIFIGKEITSMATRIX $38 \times 38$

7130711035003068, 7270726006501338 , 5120453041303528 , $311024607770776 \mathrm{~B}$. $1301130012771276 \mathrm{~B}$, 13151314010101478 . $102015010230526 \mathrm{~B}$, 1511024052704708 , 153 ' 1.

1521025053004718 , $105015310260531 \mathrm{~B}$. 2340233010601548 . $303030203010300 B$, $405040400560124 B$. $127051405050060 \mathrm{~B}$. $756072406710635 B$, 5030443040203408 , 13051304130213018 ,

$214130305250466 \mathrm{~B}$, 13271326132513248 , $1334133313320150 \mathrm{~B}$, 4700430036703258 . $1357135613551354 \mathrm{~B}$, 13641363015202178 . 5310472043203718 , $330026514051404 \mathrm{~B}$. 204

14251424142314228 , 14321431015602238 . $157022414120535 B$. 2251413053604778 . $226141405370500 \mathrm{~B}$ 1620227141505408 , $234023301630230 \mathrm{~B}$, $303030203010300 \mathrm{~B}$. 4050404012401718 . 1740533050501268 . $1374136113451330 \mathrm{~B}$, 5030443040203408 , $1133110614261425 B$. $41126505340475 \mathrm{~B}$. 12461225120311608 . $1442144114400224 \mathrm{~B}$. 4770437037603348 . 2521

12271205116211368 , $1454145302260044 \mathrm{~B}$, 5400501044104008 , 3370274125212318 , 10741073107210718 .

$1117111602050023 \mathrm{~B}$. 2060024112305178 , 251147052004618 , 261172052104628 . 2110027121405228 , 2340233021200308 . 3030302030103008 . $405040401710007 \mathrm{~B}$, 120524050501738 . 14551451144414368 , 5030443040203408 ,
640132064305108 , $351030702440714 B$. 7440743074207418 . 7600066013407308 . $513045404140353 \mathrm{~B}$, $365032302601302 B$. 2611320131713168 . 13351334133313328 , 13511350134701038

2641364136301048 , 3720330026513768 , 2400237023602358 . 3420055012303128 ,

570125041504068 . 700136101100618 13451330131210078

1160164103705428 13111310130713068 , $1316131513140147 \mathrm{~B}$. 4270366032402618 13411340133713358 . $151021613360527 \mathrm{~B}$ $370032602631360 \mathrm{~B}$ 13711370136713668 . 13760153022013658 5320473043303728

3740332026714268 , 2701435143414338 . $1443144214411440 \mathrm{~B}$, 14501447144601608 , $273145414530161 \mathrm{~B}$, 4010337027414578 , 2400237023602358 , 3420123017003318 . 1250172043404068 , $155022214100127 \mathrm{~B}$. 14441436142714068 , 1640231141705428 . $1245122412021157 \mathrm{~B}$, $1433143214310223 B$, 4360375033302708 , 11611135111014438 . 2250043126705368

$377033502721250 \mathrm{~B}$. 12061163113711128 . 14570227004512718 . 5410502044204018 . 3560314025110758 . $252112211211120 \mathrm{~B}$. 11461145114411438 . $1171117011670207 \mathrm{~B}$. 2551213121202108 . $363032102561234 B$, $240023702360235 B$, 3420170000603228 . 1720010042504068 . 2130031125501748 . 11651141111414608 2310047127305428
VP28 75 VP2B 76

VP28 77 VP28 78 VP28 79 VP28 80 VP28 BI VP2B 82 VP28 83 VP28 84 VP28 B5 VP2B 86 $\begin{array}{ll}V P 28 & 87\end{array}$ VP2B $8 B$ VP28 89 VP28 90 VP28 91 VP28 92 VP28 93 VP28 94 VP28 95 VP28 96 VP28 97 VP28 98 VP28 99 VP2B 100 VP2B 101 VP28 102 VP2A 103 VP2A 104 VP28 105 VP28 106 VP28 107 VP28 108 VP2A 109 VP2A 110 VP28 111 VP2B 112 VP2B 113 VP2R 114 VP28 115 VP2A 116 VP28 117 VP2B 118 NPPE 110 VP28 120 VP2E 121 VP2R 122 VP28 123 VP28 124 VP28 125 VP2B 126 VP28 127 VP28 128 VP2a 129 VP28 130 VP2B 131 VP28 132 VP28 133 VP2B 134 VP2A 135 IVP28 136 VP28 137 VP2A 138 VP28 139 VP28 140 VP2B 141 VP28 142 VP28 143 VP28 144 VP2B 145 VP28 i46 VP28 147 VP28 148 VP28 149 VP2 150 VP2B 151 VP2B 152 VP28 153 VP28 154 VP26 155 VP2B 156

$F_{1}=X \operatorname{XVP}(1) * Y V P(2)-X V P(2) * Y V P(1)$
$F 23=X V P(2) * Y V P(3)-X V P(3) * Y V P(2)$

$F F=F 12+F 23$

$X M=((X V P(1) * F 12+X V P(3) * F 23) / F F+X V P(2)) / 3$.

$Y M=((Y V P(1) * F 12+Y V P(3) * F 23) / F F+Y V P(2)) / 3$.

$X D P(1)=X V P(3)-X M$ s $Y D P(1)=Y V P(3)-Y M$ 
C

$X D P(Z)=-X M \quad \& \quad Y D P(Z)=-Y M$

VP28 157

$I M=1$

$M M=M(1)$

VP2B 158

VP28 159

VP28 160

DO 11 IDR $=1,4$

CALL DRPL21 $10(1), 0(232))$

VP2B 161

VP28 162

VP28 163

VP28 164

DO $12 \quad I=1,252$

$I Q=M M \cdot A N D \cdot .77778$

$Q(I Q)=Q(I Q)+D(I)$

$M M=M M / 100008$

VP28 165

VP2B 166

VP28 167

IF (MM.NE.OO) GOTO 12

$I M=I M+1$

VP28 167

VP2B 169

VP28 170

$\begin{array}{ll}\text { VP2B } & 171 \\ \text { VP2B } & 172\end{array}$

12 CONTINUE

VP2B 173

VP28 174

VP28 175

VP28 176

$c^{11}$

$X D P(2)=X V P(I D R)-X M$ S YDP (Z) $=Y V P(I D R)-Y M$

VP2B 177

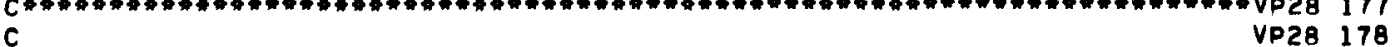

$\begin{array}{lll}\text { C KONDENSATION DER INTFRNEN FREIHEITSGRADE } & \text { VP28 } 178 \\ \text { CP28 } 179\end{array}$ C

CH*

$\begin{array}{lll}K=1 \$ K D=40 & \text { VP2B } 183\end{array}$

VP2B 185

VP28 186

$\begin{array}{rr}\text { PIVOT }=-1.1 \cap(K) & \text { VP2B } 187\end{array}$

$\begin{array}{ll}I A=K+1 & \text { VP2B } 188\end{array}$

$K=I J=K+K n \quad$ VP2B 189

$K D=K D-1 \quad$ VP2B 190

IE $=K-1 \quad$ VP2A 191

C

DO $2 \mathrm{i} \cdot \mathrm{I}=\mathrm{IA} \cdot \mathrm{IE}$

QIP $=Q$ QII)*PIVOT

DO $21 \mathrm{~J}=I, I E$

VP2B 192

VP2A 193

VP2A 194

VP2B 195

$\theta(I J)=Q(I J)+Q I P * Q(J) \quad$ VP2B 196

$21 I J=I J+1 \quad$ VP2B 197

C $1 \mathrm{~J}=1 \mathrm{l}+1.0$

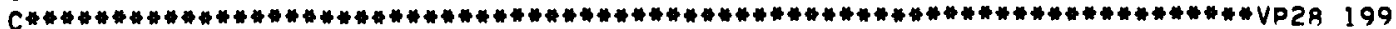

C $\quad$ VP2B 200

$\begin{array}{ll}\text { C BEHANOLUNG DER STUETZEN } & \text { VP2B } 201 \\ \text { C } & \text { VP2B } 202\end{array}$

C

CW
$C$
VP2B 204

IF(STKONST.LT.O.) GOTO $30 \quad$ VP2B 205

$\begin{array}{ll}C=C E=0 . & \text { VP28 } 207\end{array}$

$K=1$ S $K D=40 \quad$ VP2B 208

VP2B 209

$Q G=Q(K+K D-2)$ S $\quad Q E=Q(K+K D-1) \quad$ VP28 210

$C=C-Q G * 2 / D(K)$ S $C E=C E-Q G * Q E / Q(K) \quad$ VP2A 211

VP28 212

$71 \quad K D=K D-1 \quad$ VP2B 213

$Q(818)=C \quad S \quad Q(819)=C E \quad$ VP2B 215

VP28 216

$\begin{array}{ll}\text { PIVOT }=\text { C-STKONST\#FOU**2 } & \text { VP28 } 217\end{array}$

STKRG $=$ FQU*C/PIVOT \& STKRE $=$ FQU*CE/PIVOT $\quad$ VP28 218

$\begin{array}{ll}I J=155 & \text { VP2B } 219\end{array}$

$L I=189 \$ L I D=35 \quad$ VP28 220

DO $72 \quad I=1,34 \quad$ VP28 221

QIP $=-$ - (LI)/PIVOT $\quad$ VP28 222

IF (I.GT.6) STKRAFT $(I-6)=-F Q U * O I P \quad$ VP2B 223

$L J=L I$ S $L J D=L I D \quad$ VP2A 224

DO $73 \quad J=1,36 \quad$ VP28 225

$Q(I J)=Q(I J)+Q I P * Q(L J) \quad$ VP2B 226

$\begin{array}{ll}I J=I J+1 & \text { VP28 } 227\end{array}$

$L J=L J+L J D \quad$ VP28 228

$73 \quad L J D=L J D-1 \quad$ VP2B 229

$L I=L I+L I D \quad$ VP28 230

LID $=$ LID-1 V VP2B 231

C
CPD

C C

C AUSTAUSCHSCHRITTE ZUR BESTIMMUNG VON WMITTE, WMITTEG UND WMITTEE VP2B 234
VP2B 235

C AUSTAUSCHSCHRITTE ZUR BESTIMMUNG VON UMITTE, WMITTEO UND UMITTEE VP2B 236

C 


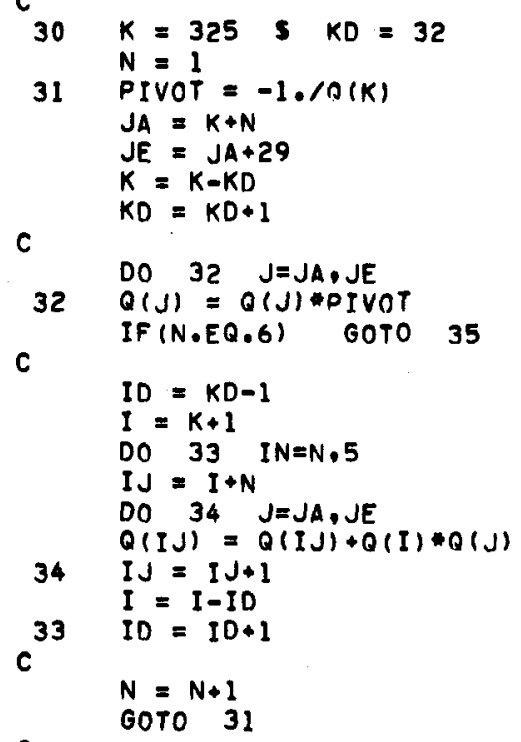




\section{SUBROUTINE VKPL24}

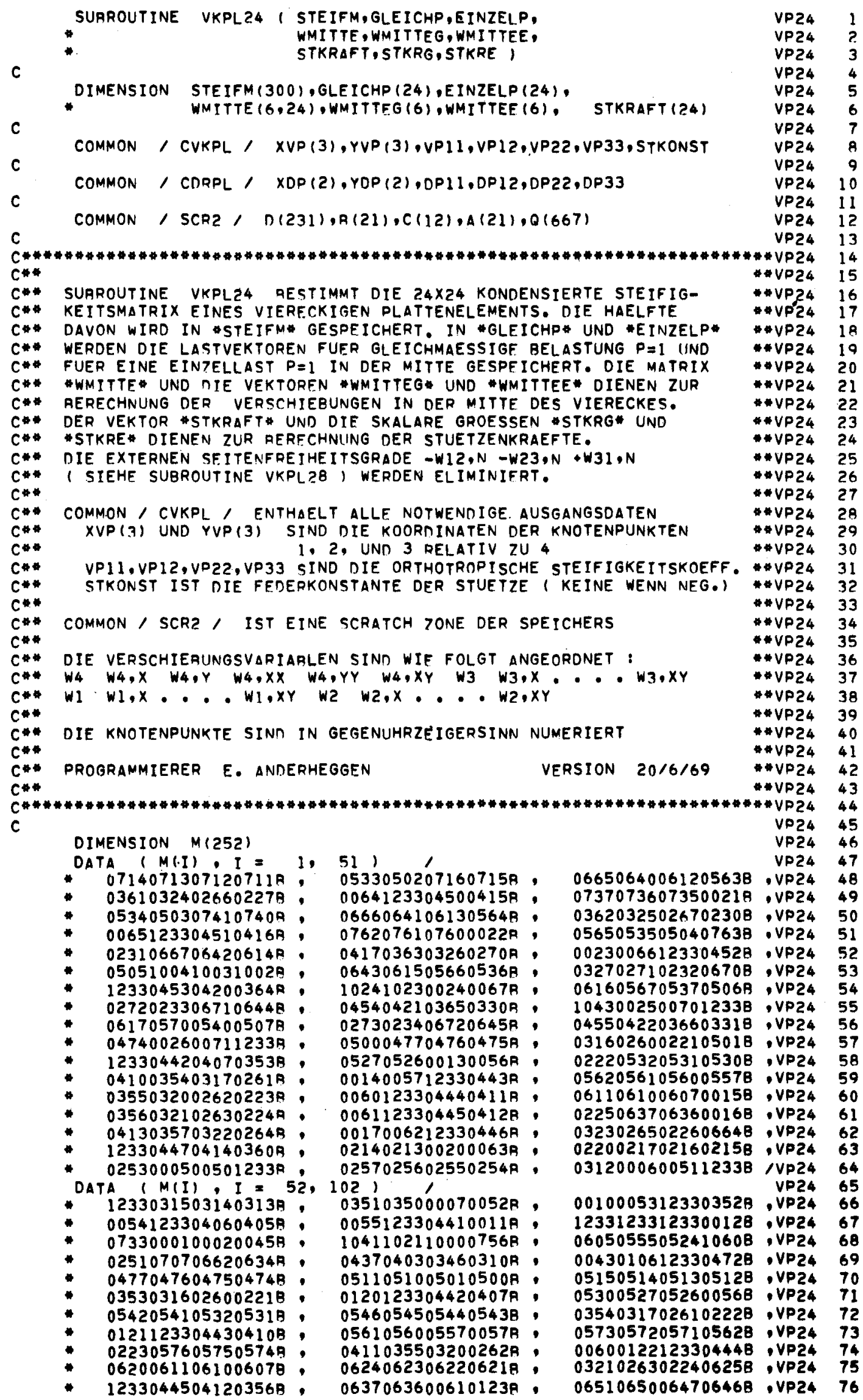


- 02640225065306528 . 06760675067406738 . 1062006301251233 A. 12330456042303678 . $0424037003330275 A$. $0371033402760237 \mathrm{~A}$

DATA I $M(I), I=103$ $0372033502770240 B$. $0427037303360300 \mathrm{R}$. $1233046304300374 \mathrm{R}$. 0253005001121233 . $^{\circ}$ $1233031503140313 P$, 01161233040604058 . 0524004500460110 . 02511165115411428 . 10651064106310629 . $0367033202740235 \mathrm{~A}$. 1106110511041103 A 01761233045704248 . 02371126112511248 . 11341133113211318 . $1233046104260372 A$. $0300024111531152 \mathrm{~A}$, 11621161116011579 . DATA $(M(I) \quad I=154$. 11670141020212338 . $1233046404310375 R$. 04320376034103039 . 03770342030402458 . 0400034303050246 , $0435040103440306 R$. 1233047104360402 , 0253011201531233 A , $1233031503140313 \mathrm{~A}$. $0157123304060405 B$. $1076011001110152 \mathrm{R}$. 02511226122312179 . 11721171117011679 . $0375034003020243 A$, 07510726120312022 . 00361233046504328 . 02451054103510159

DATA $(M(I) \quad I=205$ $0730121612151214 B^{\circ}$, $0306024710561037 A$ : $1020077707550732 A$. 07110210004212339 . 12330450041503618 . $0416036203250267 B$. $0363032602700231 \%$. $0364032702710232 R$, $0421036503300272 R$. 1233045504220366 . $025301530005.1233 R$. $1233031503140313 A$ $0011123304060405 R$. $1175015200040001 \mathrm{~A}$. C 02511060104110212 .
$0446041303570322 R$, $0265022607000677 R$. $1066106510641063 R$. $0073013512330457 R$. $0136123304600425 R$. 1531

$0137123304610426 \mathrm{~A}$. $0076014012330462 R$. $0214021300770141 R$. $0257025602550254 \mathrm{R}$. $0351035000520114 A$. $0117123304410054 \mathrm{~B}$. $0662063406050555 B$. 043704030346031 OR. $1071107010671066 \mathrm{R}$. $0175123304560423 R$. $1112111111101107 R$. $1117111611150135 R$. $0425037103340276 R$. $1140113711361135 \mathrm{~A}$. $1145114401370200 \mathrm{R}$, $0462042703730336 \mathrm{~A}$. $0301024211641163 A$. 204 )

11731172117111700 , $1200117701420203 \mathrm{~A}$. 0143020412330465 A , $0205123304660433 R$, $0206123304670434 \mathrm{R}$. $0146020712330470 R$. 02140213014702108 . $0257025602550254 \mathrm{~A}$. $0351035001140155 \mathrm{~A}$. 01601233044101168 . $1154114211271113 \mathrm{~A}$. $0437040303460310 A$. $0750072511741173 R$. $0035123304640431 \mathrm{~A}$ : $1053103410140773 A$. $1210120712060204 R$. 04330377034203048 ? 2521

$1036101607750753 \mathrm{~B}$. 12221221020600408 $0470043504010344 R$; $0307025010571040 R$, $0715071407130712 R$. $0736073501670021 \mathrm{~B}$. $0170002212330451 \mathrm{~A}$. $0023123304520417 \mathrm{~A}$. $0024123304530420 R$. $0173002512330454 \mathrm{R}$ ? $0214021301740026 R$ ? $0257025602550254 R$, $0351035001550007 R$. $0012123304410157 A$. $1223121712121204 R$. $0437040303460310 \mathrm{R}$. $1101110000720134 \mathrm{R}$,

$0664006201241233 R$.VP24 $04470414036003238 . V P 24$ 03320274023510678 ,VP24 02361104110311028 ,VP24 11201117111611158 .VP24 11331132113100748 /VP24 VP24

$0241114511440075 B, V P 24$ 03370301024211568, VP2 $0220021702160215 B$,VP2 03120051011312338 .VP24 $00530115123303528, V P 24$ 12331233123300558 .VP2 11271113107607078 ,VP24 01060150123304728 ,VP24 10751074107310728, VP24 1102110111000134 ,VP24 03700333027502368 .VP24 $1123112211211120 B$,VP24 01360177123304608 .VP2 96 03350277024011418 ,VP24 97 11511150114711468 , VP24 98 11560140020112338, VP24 99 04630430037403378 /VP24 100 VP24 101

03400302024311748, VP24 102 $02441203120212018, V 024103$ 12111210120712068 ,VP24 104 12161215121401448 ,VP24 105 $02471222122101458, V P 24106$ 03450307025012258 ,VP24 107 02200217021602158, VP24 108 $03120113015412338, V P 24109$ 01150156123303528, VP2 110 12331233123301178 ,VP24 111 1212120411751165 . VP24 112 01500211123304728 ,VP24 113 10521033101307728, VP24 114 12011200117702038 .VP24 115 $0376034103030244 A$,VP24 116 07740752072712118 ,VP24 117 02050037123304668 /VP24 118 VP24 110

03430305024610558 ,VP24 120 10170776075407318, VP24 121 12250207004112338 ,VP24 122 0471043604020345 , VP24 123 0324026602270716 , VP24 124 02300741074007378 .VP24 125 07630762076107608, VP24 126 10041003100201718 ,VP24 127 02331024102301728 .VP24 128 $0331027302341043 B$.VP24 129 02200217021602158 ,VP24 130 03120154000612338 ,VP24 131 01560010123303528 .VP24 132 12331233123301608 ,VP24 133 10000756073312268 ,VP2 134 $0211004312330472 B$ /VP24 135 VP24 136

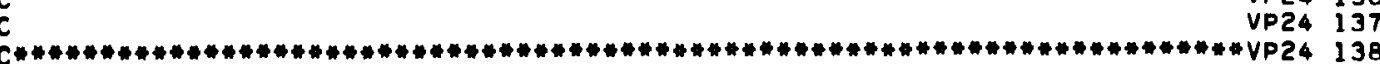
C BILOUNG DER NICHT KONDENSIERTEN STEIFIGKEITSMATRIX $38 \times 38 \quad$ VP24 140 VP24 141

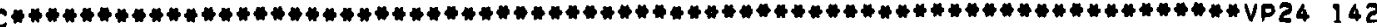
VP2 145 DP11 $=$ VP11 \& OP12 $=$ VP12 \& DP22 = VP22 \& DP33 $=$ VP33 VP24 144 $0011=1,667$

$O(I)=0$.

$Q(1>0)=-1$

C

$F 12=X V P(1) * Y V P(2)-X V P(2) * Y V P(1)$

$F 23=X V P(2) * Y V P(3)-X V P(3) * Y V P(2)$

$F F=F 12+F 23$

$X M=((X V P(1) * F 12+X V P(3) * F 23) / F F+X V P(2)) / 3$.

$Y M=((Y V P(1) * F 12+Y V P(3) * F 23) / F F+Y V P(2)) / 3$.

C

$X D P(1)=X V P(3)-X M \quad S \quad Y D P(1)=Y V P(3)-Y M$

$X D P(2)=-X M$ S $Y O P(2)=-Y M$

IM $=1$

$M M=M(1)$

$C(1)=C(7)=0$.
VP24 145

VP24 146

VP24 147 VP24 148 VP24 149 VP24 150 VP24 151 VP24 152 VP24 153 VP24 154 VP2 4155 VP24 156 VP24 157 VP24 158 VP24 159 
C

CALL DRPL?I $10(1), R) \quad$ VP24 161

C

VP24 163

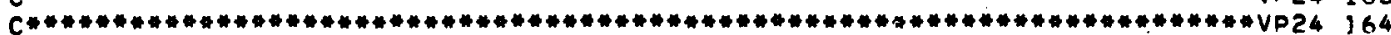

$\begin{array}{llll}C & & \text { VP24 } & 165 \\ C & \text { ELIMINATION DER EXTEREN FREIHEITSGRADE W,N } & \text { VP24 } 166\end{array}$

C

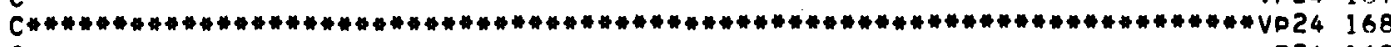

c

$D X=X D P(2)-X D P(1)$ S $D Y=Y D P(2)-Y D P(1)$

VP24 169

$D X=X D P(2)-X D P(1)$
$S=-S Q R T(D X * D X+D Y * D Y)$

$C(2)=C(8)=-.5 * D Y / S$

$c(3)=c(9)=-5 * n \times / 5$

$C(4)=.5 * 5 * C(2) * C(3)$

$C(5)=c(10)$

$C(6)=.25 *(n y * C(2)+D x * C(3))$ s $\quad C(12)=-C(6)$

VP24 170

VP24 171

VP24 172

VP24 173

VP24 174

VP24 175

VP24 176

VP24 177

VP24 178

$00 \quad 50 \quad I=1.19$

$\begin{array}{lll}\text { VP2 } & 178 \\ \text { VP24 } & 179\end{array}$

VP24 180

$K=K+K D$ S $K D=K D-1$

50 CONTINUE

$A(20)=D(227) \quad \$ \quad A(21)=D(228)$

$K_{0}=\frac{1}{51} \quad 1=1,12$

$C I=C(I)$ S $A I=A(I)$

DO $52 \mathrm{~J}=1.12$

$D(K)=D(K)+C I * A(J)+C(J) * A I+C I * C(J) * A(19)$

$52 \quad K=K+1$

DO $53 \mathrm{~J}=13,21$

$D(K)=D(K)+C I * A(J)$

$53 \quad K=K+1$

$51 \quad B(I)=B(I)+C I * A(19)$

c

DO $12 \quad I=1.252$

$I O=M M \cdot A N D \cdot 7777 B$

$Q(10)=O(10)+D(I)$

$M M=M M / 10000 R$

IF (MM.NE.0) GOTO 12

$I M=I M+1$

VP24 IRI

VP24 182

VP24 IB3

VP24 184

VP24 185

VP24 186

VP24 187

VP24 188

VP24 IR9

VP24 190

$\begin{array}{lll}V P 24 & 191\end{array}$

VP24 192

$\begin{array}{lll}\text { VP2 } & 193\end{array}$

VP24 194

VP24 195

VP24 196

VP24 197

VP24 19R

VD24 199

VP24 200

VP24 201

$M M=M(I M)$

VP24 202

VP24 203

VP24 204

VP24 205

VP24 206

VP24 207

$\begin{array}{ll}X D P(1)=X D P(2) \quad \$ \quad Y D P(I)=Y D P(2) \\ X D P(2)=X V P(I D R)-X M & S \quad Y D P(2)=Y V P(I D R)-Y M\end{array}$

C

C

KONDENSATION DFR INTERNEN FREIHEITSGRADE

VP24 2 R

$V P 24209$

VP24 210

VP24 211

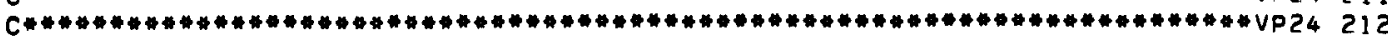

C

$K=1$ S $K D=36$

DO $21 \quad N=1,10$

PIVOT $=-1 \cdot 1 \cap(K)$

$I A=K+1$

$K=I J=K+K n$

$K D=K D-1$

$I E=\mathrm{K}-1$

$V D 24 \quad 213$

VP24 214

VP24 215

VP2 2216

VP24 217

VP24 218

VP24 219

VP24 220

C

DO 21 I I IA,IE

QIP $=$ Q(I) MPIVOT

DO $21 \mathrm{~J}=\mathrm{I}, \mathrm{IE}$

VP24 221

VP2 222

VP24 223

VP24 224

$0(I J)=0(I J)+0 I P * 0(J) \quad$ VPS4 252

$21 I J=I J+1 \quad$ VP24 226

C

$\begin{array}{rr}C & \text { VP } 24228 \\ C & \text { VP24 } 229\end{array}$

$\begin{array}{lr}C & \text { BEHANDLUNG DER STUETZEN } \\ \text { C VP24 } 230\end{array}$

VP24 231

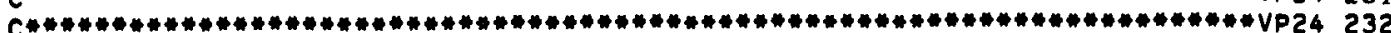

VP24 233

IF(STKONST.LT.0.) GOTO $30 \quad$ VP24 23

C

$C=C E=0$.

$K=1$ S $K D=36$

DO $71 \quad N=1,10$

$Q G=O(K+K D-2)$ S $O E=Q(K+K D-1)$

$C=C-O Q * 2 / D(K)$ S $C E=C E-Q Q * Q E / Q(K)$

VP24 235

VP24 236

VP24 237

VP. 24238

VP24 239

VP24 240

VP24 241

$K=K+K D$

VP24 242

$71 \quad K D=K D-1$ 


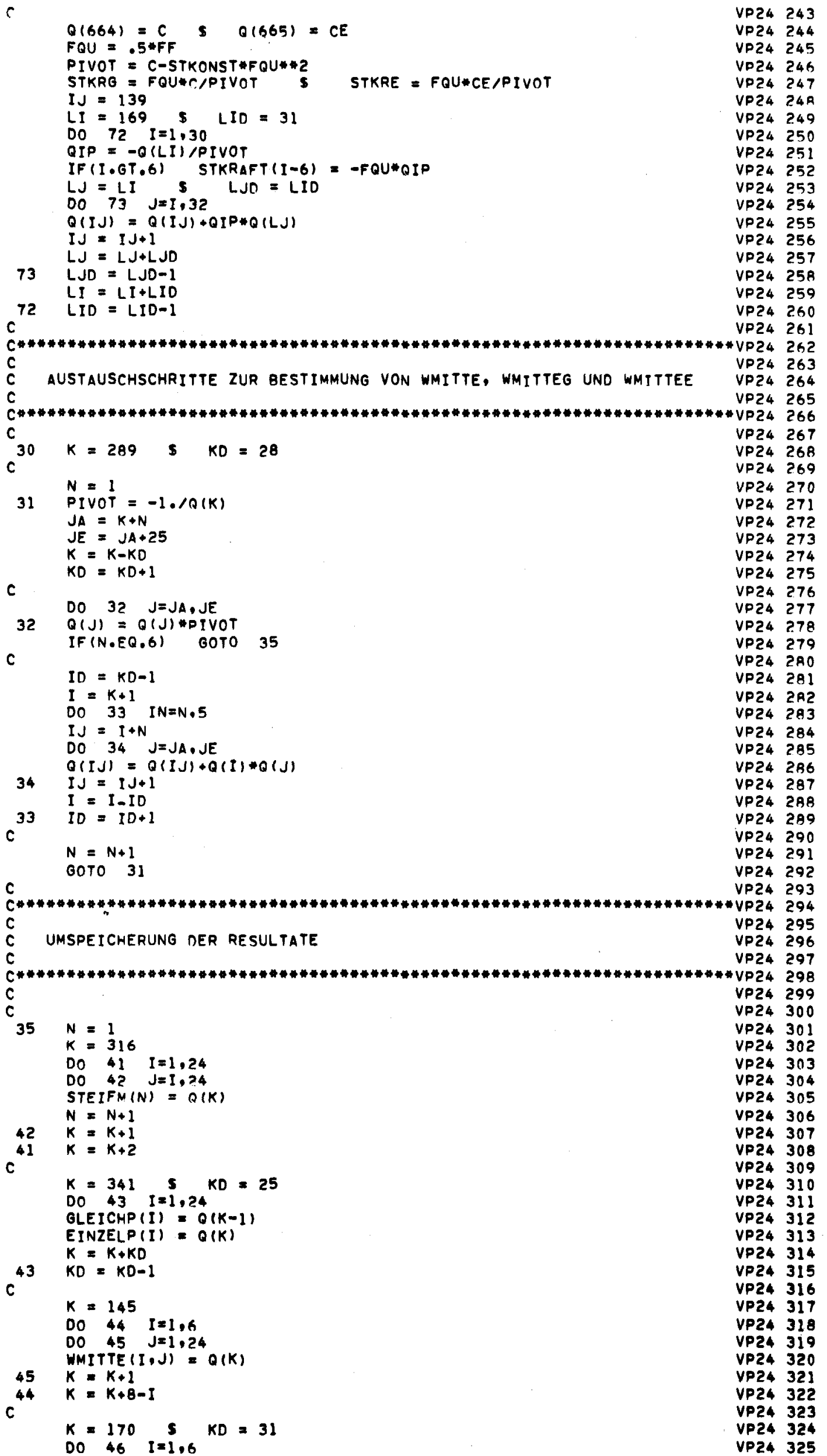




\section{SUBROUTINE VKSC24}

c

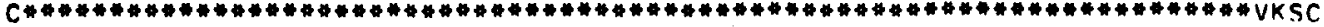

C.*

$C * *$

C**

$C * *$

C**

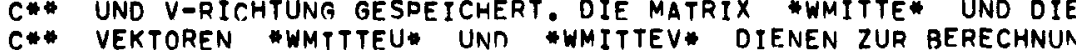

C* VERSCHIERUNGEN IN DER MITTE DES VIFRECKES.

$C * *$

$C * *$

C*

$C * *$

$C * *$

$C *$

$C * *$

C** DIE VERSCHIEBUNGSVAR

C. V4 U4,X V4,Y $(114, Y+V 4, X)$

U3 V3 U3,X V3,Y $\quad(U 3, Y+V 3, X)$

C* UI VI UI,X VI,Y $(U 1, Y+V I, X)$

C* U2 V2 U2,X V?,Y $(U 2, Y+V Z, X)$

$C * *$

$C *$

$C *$

C**

DIE KNOTENPUNKTE SINC IN GEGENUHRZEIGERSINN NUMERIERT.

C

COMMON / CORSC / XDS (2),YOS (2).0S11.0512.0522.0533

EQUIVALENCE (XOS,XDS $),(X D S(2), X D S 2),(Y D S, Y D S 1),(Y D S(2), Y D S 2)$

DIMENSION M(?.07)

DATA I $M(I) \cdot I=1.54$ )

- $0502050105000477 R$.

- 0147011200540015 .

- $0427040103520322 R$.

- 02720551055005478

05700240020501518

$0152011500570020 A$.

00600021045704328

- $0022046004330405 B$.

$0007026702660265 R$.

$0047001003200317 R$.

01430106005000119 .

$0424023302000144 \mathrm{P}$

$0146011100530014 \mathrm{~B}$

$0044004300420041 A$.

$0521022701740173 \mathrm{~B}$

$0037047504500422 \mathrm{~A}$

- 0313064706300610 .

- $0262026102260172 A$

DATA ( $M(I), I=55,109$

* 00070303030203018 .

- 03300327032003178 .

- 03460345023101768.

- 0143010600500011R. $0524052302360203 B, 0271052705260525 B$. VKSC $0113005500160454 B, 0546023702040150 B, V K S C$ $0430040203530323 B, 0114005600170455 B$, VKSC $0324027305720571 B, 0456043104030354 B$, VKSC $0612061102410206 B ; 0404035503250274 B, V K S C$ $0242020701530116 B, 03560326027506318$, VKSC $0210015401170061 B$. $0264026302620243 B$, VKSC 01750141010400468 . 03160315031402308 . VKSC $0231017601420105 B$ : $0350034703460345 B$. VKSC $0376037502320177 \mathrm{~B}: 0107005100120377 \mathrm{~B}$. VKSC 01100052001304258,04520234020101458 , VKSC 00020001023502028,00060005000400038 . VKSC 01020101010000458,01400137013601038, VKSC 06270607056605448,03730343031206468 , VKSC 02250171013400768,05670545052202608 . VKSC 04510423037403448,01350077004004768 , VKSC 60 02660265026402638 .

01750141010400468 03340333033203318 . 03600357035003478 03760375023201778 . 03000277027602678 , VKSC 61 03160315031402308 , VKSC 63 01420105004700108 , VKSC 64 $0364036303620361 \mathrm{~B}$, VKSC 65 04100407040603778 , VKSC 66 
- 00120413041204118 - 04400437043604358 . - 04640463046204618 . - 06530652065106508 - 0671067006670666B - 00250706070507048 . - $0160012300650026 \mathrm{~B}$ - 00300744025002158 . - 0041000600050004A. - $0173014001370136 \mathrm{~A}$.

- 0730071507010664 A - $0423037403440313 B$. - $0172013500770040 R$ ? - 0661066006570656R. DATA I M(I) I I =100,162

- 0671067006670666A. - $0212015601210063 B$. - $0025071407130712 B$ - $0725072407230722 \mathrm{~A}$ - $0735073407330732 R$. - 07450744025002158 . - $0755025102160162 R$ - $0765025202170163 R$. - $0775077402530220 \mathrm{~B}$. - 0034100410031002R - $0223016701320074 \mathrm{~B}$ - $0004000300020001 A$. - 0136010301020101B. - $0763075307420730 \mathrm{~B}$. - $0665026002250171 R$. - 0040101510121006B. - 0513076207610760R - 07650252021701638 DATA I M(I),I $=163,207$ - 0071003206410622B. - $0603056205400515 \mathrm{~A}$. $0516100410031002 \mathrm{R}$, - $1007025502220166 R$. - $0223016701320074 \mathrm{R}$ - $0170013300750036 \mathrm{~A}$ - $0147011200540015 A$ - $0204015001130055 \mathrm{~B}$. - 05700240020501518 - 00600021061206112 . - 0001024302100154R. - $0101010000450044 R$. - 10051000077207638 - $0171013400760037 B$. C - $0610056705450522 B$

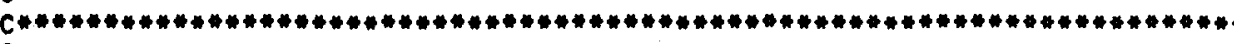
C

21

BILDUNG DER NICHT KONOENSIERTEN STEIFIGKEITSMATRIX $30 \times 30$

02000144010700518 01100052001304418 $0053001404660465 B$ 00620023063506548 01210063002406728 02130157012200648 , 07330732024702148 02160162012500678 $0045004400430042 B$ $0343031202270174 B$ 00760037075307428 07020665047604518 06510650026102268 . 00620023066306628 06750674067306728 . $0705070407030245 B$. $0213015701220064 B$ 00650026072707268 $0741074007370736 \mathrm{~B}$ $0751075007470746 B$ 07610760075707568 $0771077007670766 \mathrm{~B}$ 00720033077707768 02220166013100738 00750036101302568 $0042004100060005 B$ 01740173014001378 . 10111005100007728 $0743073107160702 B$ 02260172013500778 06210601056005368 . 07710770076707668 02530220016401278 . 00720033064206238 . 06240604056305418 . 05640542051710108 . 05430520101302568 . 05000477025702248 05240523023602038 . $0550054705460237 \mathrm{~B}$. 00570020057205718 . 02420207015301168 00050004000300028 01370136010301028 05440521101410118 . 07730764026002258 $0077004006470630 B$.

04340425042402338 , VKSC 04520234020101458 ? VKSC 68 $0235020201460111 B$. VKSC 69 02440211015501208 , VKSC 70 0703024502120.1568 , VKSC 71 07210720071702468 , VKSC 72 $0161012400660027 B$, VKSC 73 00030002000102518 , VKSC 74 $0103010201010100 B$, VKSC 75 $0475045004220373 B$, VKSC 76 $0260022501710134 B$, VKSC 77 07540743073107168 , VKSC 78 06550654065306528 , VKSC 79 02440211015501208 / VKSC 80

00240700067706768 $0711071007070706 B$, VKSC 83 07210720071702468 , VKSC B4 02470214016001238 , VKSC 85 $0161012400660027 B$, VKSC 86 $0125006700300752 B$, VKSC B7 $0126007000310762 B$, VKSC BA $0164012700710032 B$. VKSC A9 02540221016501308 . VKSC 90 $0035101010070255 B$, VKSC 91 $0257022401700133 B$, VKSC $0100004500440043 B$. VKSC 93 07150701066402278 , VKSC 94 $0134007600371014 B$, VKSC 95 $1001077307640754 B$. VKSC 96 $0757075607550261 B$, VKSC 97 $0126007000310640 B$, VKSC 98 $0602056105390514 \mathrm{~B}$, VKSC 09 VKSC 100 $0777077607750774 A$, VKSC 101 $0254022101650130 B$. VKSC 102 01310073003406438 , VKSC 103 $0035064406250605 B$, VKSC 104 $0645062606060565 \mathrm{~B}$, VKSC 105 05040503050205018 . VKSC 106 $0016052705260525 B$, VKSC 107 01140056001705518 , VKSC 108 02410206015201158 . VKSC 109 01170061002206318 , VKSC 110 00430042004100068 . VKSC 111 02270174017301408 , VKSC 112 06460627060705668, VKSC 113 10151012100610018 , VKSC 114 02610226017201358 , VKSC 115 VKSC 116 VKSC 117 VKSC 118 VKSC 119 VKSC 120 VKSC 121 VKSC 122 VKSC 123 DS11 = VS11 S DS12 = VS12 S DS22 = VS22 S $0533=$ VS33 VKSC 124 DO $21 I=1.52 \mathrm{~A} \quad$ VKSC 125 $Q(I)=0$.

$F_{12}=X V S(1) * Y V S(2)-X V S(2)$ *YVS $(1)$

$F 23=X V S(2)$ HVS $(3)-X V S(3)$ HVS $(2)$

$F F O S=F 12+F 23$

$X M=($ XVS $(1) * F 12+X V S(3) * F 23) / F F O S+X V S(2)) / 3$

$Y M=(1 Y V S(1)$ \#F12+YVS(3)*F23)/FFOS+YVS(2))/3.

$X D S 1=X V S(3)-X M$ S YDS1 $=$ YVS(3)-YM

$X D S 2=-X M \quad Y$ S YDS2 $=-Y M$

$I M=1$ s $M M=M(1)$

c

DO 24 IDR=1,4

CALL DRSC1810(1),D(172),D(190)1

DO 23 KD=1.207

KO = MM.AND.77778

$Q(K O)=Q(K O)+D(K O)$

$M M=M M / 10000 B$ S IF(MM.NE.0) G0TO 23

$I M=I M+1$ S $M M=M(I M)$

CONTINUE

XOS1 $=$ XOS2 $S \quad X 052=X V S(I D R)-X M$

YOS1 = YDS2 S YDS2 = YVS (IOR) $-Y M$

24

CONTINUE
VKSC 126

VKSC 127

VKSC 128

VKSC 129

VKSC 130

VKSC 131

VKSC 132

VKSC 133

VKSC 134

VKSC 135

VKSC 136

VKSC 137

VKSC I38

VKSC 139

VKSC 140

VKSC 141

VKSC 142

VKSC 143

VKSC 144

VKSC 145

VKSC 146

VKSC 147

VKSC 148

VKSC 149 


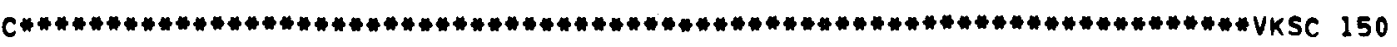

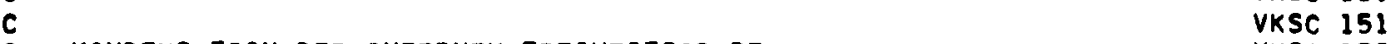

C KONDENSATION DER INTERNEN FREIHEITSGRADE VKSC 152

C

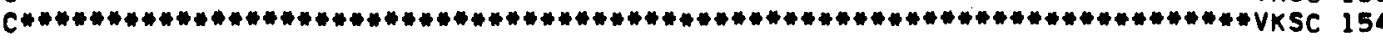

$K=1$ S KO $=32$

DO $31 \mathrm{~N}=1,6$

PIVOT $=-1 . / 0(K)$

$I A=K+1$

$K=I J=K+K n$

$K D=K O-1$

$I E=K=1$

DO 31 I=IA,IF

QIP $=Q(I) * P I V O T$

DO $31 \mathrm{~J}=\mathrm{I}$, IE

$\theta(I J)=Q(I J)+Q I P * Q(J)$

$I J=I J+1$

31 CONTINUE

VKSC 155

VKSC 156

VKSC 157

VKSC 158

VKSC 159

VKSC 160

VKSC 161

VKSC 162

VKSC 163

VKSC 164

VKSC 165

VKSC 166

VKSC 167

VKSC 168

C AUSTAUSCHSCHRITTE ZUR BESTIMMUNG VON WMITTE. WMITTEU UND WMITTEV VKSC 171

C

$\begin{array}{ll}C & \text { VKSC } 175\end{array}$

$K=151 \mathrm{~S} K D=2 B$

VKSC 176

VKSC 177

VKSC 178

PIVOT $=-1 \cdot / 0(K)$

$\mathrm{JA}=K+N$ S JE $=J \Delta+25$

VKSC 179

$K=K-K D \& K D=K D+1$

VKSC 1 AO

VKSC 181

VKSC 182

$Q(J)=Q(J) * P$ IVOT

IF (N.EQ.6) GOTO 45

$I D=K D-1$ \$ $I=K+1$

DO 44 IN $=N, 5$

$I J=I+N$

DO $43 \mathrm{~J}=\mathrm{JA}, \mathrm{JF}$

$Q(I J)=Q(I J)+Q(1) * \theta(J)$

$43 \quad I J=I J+1$

$I=I-I D$

$44 \quad I 0=I D+1$

$N=N+1$

GOTO 41

45 CONTINUE

VKSC 183

VKSC 184

VKSC 185

VKSC 186

VKSC 187

VKSC 186

VKSC 189

VKSC 190

VKSC 191

VKSC 192

VKSC 193

VKSC 194 VKSC 195

C

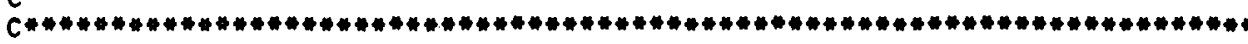

UMSPEICHERUNG NER RESULTATE

VKSC 197

VKSC 198 VKSC 199

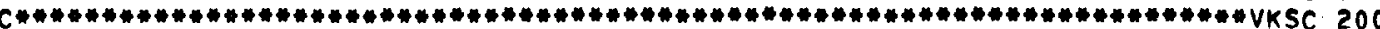

$N=15 K=178$

DO $52 I=1.24$

DO $51 \mathrm{~J}=\mathrm{I}, 24$

$\operatorname{STEIFM(N)}=A(K)$

$N=N+1$

$51 \quad K=K+1$

$52 \quad K=K+2$

$K=203 \$ K D=25$

DO $53 I=1.24$

GLEICHU(I) $\approx 0(K-1)$

GLEICHV (I) $=O(K)$

$K D=K D-1$

$K=7$

DO $55 \quad I=1,6$

DO $54 \mathrm{~J}=1.24$

WMITTE $(I, J)=\theta(K)$

$54 \quad K=K+1$

$55 \quad K=K+8-I$

$K=32 s K O=31$

DO $56 \quad I=1.6$

WMITTEU (I) $=-Q(K-1)$

WMITTEV II $=-Q(K)$

$K=K+K D$

$56 \quad K D=K D-1$

VKSC 201
VKSC 202

VKSC 203

VKSC 204

VKSC 205

VKSC 206

VKSC 207

VKSC 208

VKSC 209

VKSC 210

VKSC 211

VKSC 212

VKSC 213

VKSC 214

VKSC 215

VKSC 216

VKSC 217

VKSC 218

VKSC 219

VKSC 220

VKSC 221

VKSC 222

VKSC 223

VKSC 224

VKSC 225

VKSC 226

VKSC 227

VKSC 228

RETURN

END

VKSC 229

VKSC 230

VKSC 231 


\section{SUBROUTINE BANDMAT}

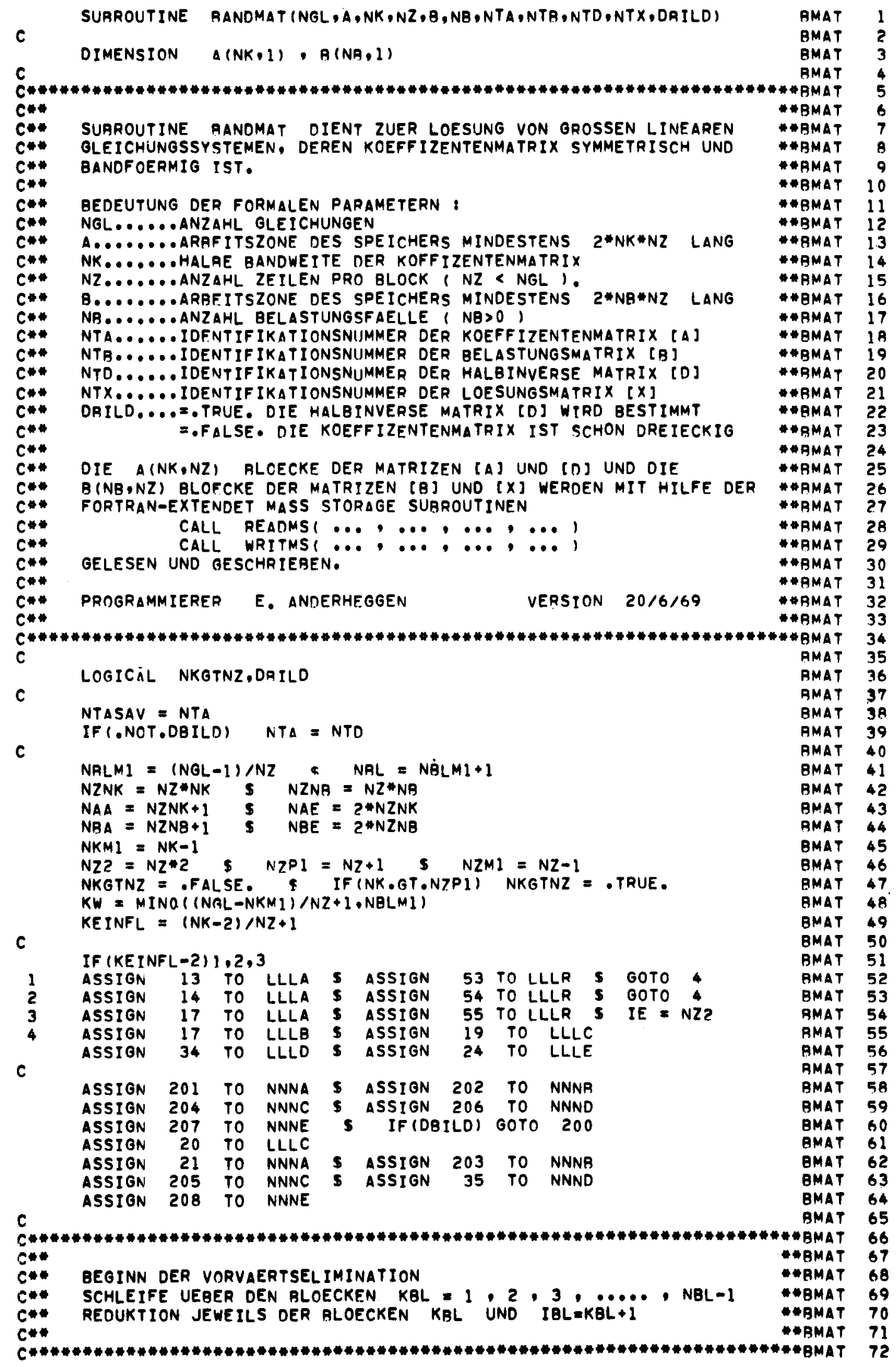


$200 \quad K E=N Z$

C

$\mathrm{KBL}=1$

100

$101 I A L=K B L+1$

$K A L M 1=K B L-1$

IF (KBL.LT.KW) GOTO 11 TO LLLC

JEK = NGL-KBLMI*NZ

IF (KBL.LT.NALMI) GOTO 11

$I E=I E E=J F K$

$K E=I E-1$

ASSIGN 16 TO LLLA

ASSIGN 41 TO LLLE $\$$ IF(DBILD) ASSIGN 40 TO LLLE

11

DO $12 K=1, K E$

PIVOT $=1 . / A(1, K)$

$I A=K+1$

GOTO LLLA, $(13,14,17)$

$I E=K+N K M I \$$ SOTO 15

$I E=M I N O(K+N K M I, N Z 2)$

GOTO LLLB, $(16,17)$

$I E=M I N O(I E, I E E)$

$I I=I-K$

GOTO LLLC. $(18,19,20)$

$J E=M I N O(N K M), J E K-K)$ S GOTO 20

$J E=N K M 1$

DO $21 \quad I=I A \cdot I E$

$A I P=A(I+I I, K)$ \#IVOT

IF (AIP.EQ.0.) GOTO 21

DO $22 J=1$, NA

$B(J, I)=B(J, I)-A I P * B(J, K)$

22 CONTINUE

GOTO NNNA, $(201,21)$

$501 \mathrm{JJ}=I-K$

$0023 \mathrm{~J}=1$, JE

$A(J, I)=A(J, I)-A I P \# A(J+J J, K)$

CONTINUE

$21 \quad J E=J E-1$

12 CONTINUE

202

GOTO NNNB, $(202,203)$

203

GOTO LLLE, $(24,40,41)$

24 IF (NKGTNZ) GOTO 30

DO $26 \quad N=1, N 2 N K$

$A(N)=A(N+N 7 N K)$

DO $27 \quad N=1, N 2 N B$

$27 \quad B(N)=A(N+N Z N B)$

$K B L=K B L+1$

BOTO 101

CALL WRITMS (NTD,A $(1,1)$, NZNK,KAL)

CALL WRITMS (NTX,B $(1,1), N Z N B, K A L)$

CALL READMS (NTA1,A $(1,1), N Z N K, K R L)$

CALL READMS (NTB1,A $(1,1)$, NZNB,KRL)

CALL READMS (NTAZ,A (NAA, I), NZNK, IBL)

CALL READMS (NTB2, R(NBA,I), NZNA, IBL)

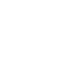

5

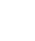
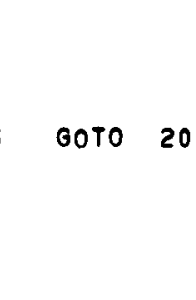

C

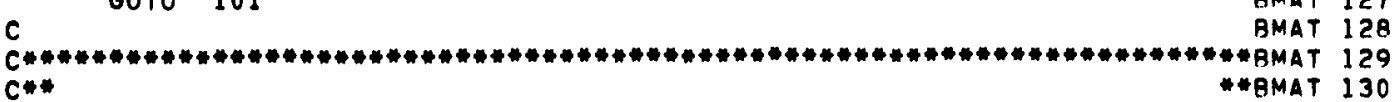

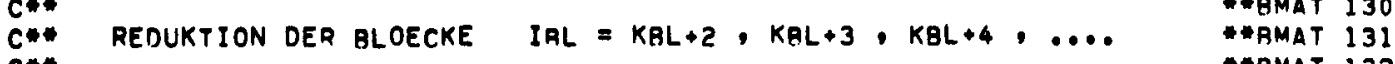

C*

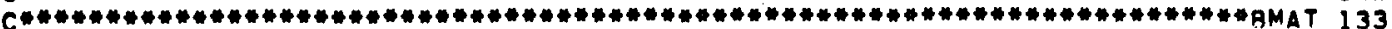

(204,205)

CALL WRITMS (NTD, A (NAA, I), NZNK, IBL)

BMAT 135

BMAT 137

BMAT 138

CALL READMS (NTA3,A (NAA,I),NZNK, IBL) BMAT 139
CALL READMS(NTB3,P(NBA,I),NZNA,IBL) BMAT 140

CALL READMS (NTA3,A (NAA,I),NZNK,IBL)
CALL READMS(NTB3,P(NBA,I),NZNA,IBL) 139
BMAT 140

IMKBL $=I B L-K B L$

BMAT 142

BMAT 143

BMAT 144

BMAT 145

BMAT 146

BMAT 147

BMAT 148

BMAT 149

BMAT 150

BMAT 151

C

JJJJ $=$ IMKNZ-NZ

BMAT 152

DO $32 K=K A, N Z$

BMAT 153

JE JEK $+K$ 
GOTO LLLD, $(33,34)$

RMAT 155

$J E=M I N O(J E$, JEMAX)

$34 \quad \operatorname{IEN}=\operatorname{MINO}(J F, N Z)+N Z$

$J J J=J J J J-K$

$I I=I I I-K$

DO $35 \quad I=N Z P], I E N$

$A I P=A(I+I I, K) * P I V \cap T$

IF (AIP.EO.O.) GCTO 35

DO $36 \quad J=1, N A$

$B(J, I)=B(J, I)-A I P * B(J, K)$

CONTINUE

GOTO NNND, (2n6,35)

$506 \mathrm{JJ}=\mathrm{JJJ}+\mathrm{I}$

DO $37 \mathrm{~J}=1$, JE

$A(J, I)=A(J, I)-A I P * A(J+J J, K)$

CONTINUE

$35 \mathrm{JF}=\mathrm{JE}-1$

CONTINUE

GOTO NNNE, $(207,208)$

IF (IMKBL.LT.KEINFL.AND.IBL.LT.NAL) GOTO 31

CALL WRITMS (NTD, A (NAA, I), NZNK, IBL)

NTAL $=$ NTAZ $=$ NTA3 $=$ NTD \& NTA1 $=$ NTB2 $=N$ TB3 $=$ NTX

$K A L=K A L+1$

C

GOTO 100

,

AMAT 156

AMAT 157

BMAT $15 B$

АMAT 159

RMAT 160

AMAT 161

BMAT IGZ

BMAT 163

RMAT 164

AMAT 165

AMAT 166

BMAT 167

BMAT 168

BMAT 169

BMAT 170

RMAT 171

BMAT 172

BMAT 173

BMAT 174

BMAT 175

AMAT 176

BMAT 177

RMAT 178

BMAT 179

BMAT 180

AMAT 191

คMAT 192

BMAT 193

40

CALL WRITMS (NTD,A (NAA, I), NZNK, NBL)

RMAT 194

PMAT 195

BMAT 196

CALL WRITMS (NTX,B (NBA, 1),NZNB,NBL)

C * ZURUECKEINSETZEN JEWEILS IN DEN A-BLOECKEN KRL UND IRL=KBL-1

SCHLETFE UERFR DEN PLOECKEN KBL = NAL, NBL-1, ...,?

C**

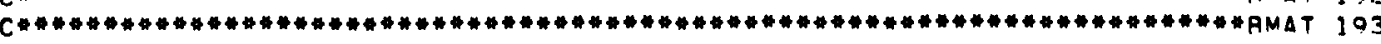

C

$K K F=N G L-N R L M I * N Z$
$K K K=K K E+N Z P I$

RMAT 194

RMAT 195

RMAT 196

BMAT 197

$I A=1$

ASSIGN 57 TO LLLS

AMAT 198

$K R L=N R L$

C

$I B L=N B L M I$

500 IF (KAL.GT.2) GOTO 51

$K K F$. $=N Z 2-1$

$K K K=K K E+2$

ASSIGN 90 TO LLLS

ASSIGN 54 TO LLLR

DO $52 \quad K K=1, K K E$

$K=K K K-K K$

PIVOT $=-1 \cdot / A(1, K)$

C

GOTO LLLR, $(5,3,54,55)$

53 IA $=K-N K M I$ S GOTO 55

$54 \quad I A=M A X O(I, K-N K M 1)$

55 IE $=K-1$

$I I=K+I$

DO $56 \quad J=1, N B$

$B(J, K)=B J P=B(J, K) * P I V O T$

DO 56 I I IA, IE

$B(J, I)=B(J, I)+A(I I-I, I) * R J P$

56 CONTINUE

52 CONTINUE

GOTO LLLS, $(57,58,90)$

CALL WRITMS (NTX,B (NBA, I),NZNB,KBL)

BMAT 199

BMAT 200

RMAT 201

RMAT 202

BMAT 203

BMAT 204

BMAT 205

BMAT 206

BMAT 207

BMAT 20A

BMAT 209

BMAT 210

RMAT 211

BMAT 212

BMAT 213

BMAT 214

AMAT 215

BMAT 216

BMAT 217

BMAT 218

RMAT 219

BMAT 220

BMAT 221

BMAT 222

BMAT 223

ВMAT 224

BMAT 225

BMAT 226

BMAT 227

BMAT 228

BMAT 229

BMAT 230

BMAT 231

BMAT 232

BMAT 233

BMAT 234

BMAT 235

c

$B(N+N Z N B)=R(N)$

$K B L=K B L-1$

GOTO 501

BMAT 236 


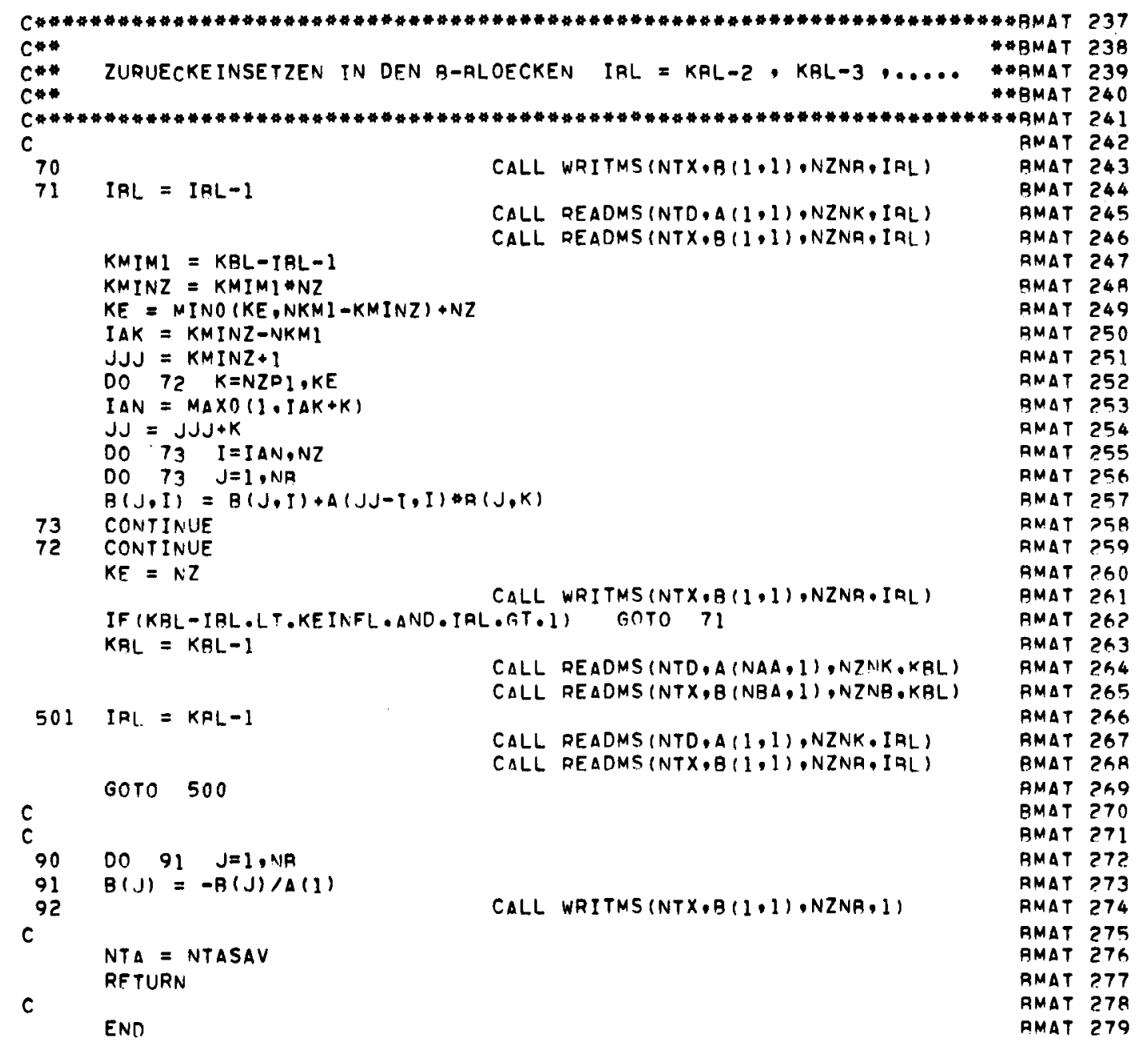

\section{SUBROUTINE BMRESID}

SURROUTINE RMRESID(NGL,A,NK,NZ,B,NR,NTA,NTB,NTX,NTR,RLAENGE)

DIMENSION A(NK, 1),R(NA, 1),RLAENGE (NB) RRES ARES

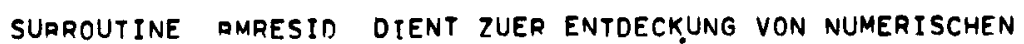

BALRN DEI DER LOESUNG VON GLEICHUNGSSYSTFME MIT DER SURROUTINE \#RRES \& SOWIE DIE LAENGEN DER RESINUENVEKTOREN DIVIOIERT DURCH DIE ARPES 10

C* LaENGEN DER ENTSPRECHENDEN LASTVEKTOREN. DIESE WERTE WERDEN IN *\#RES 11

C** "RLAENGE* GESPEICHERT.

C*: REDEUTUNG DER FORMALEN PARAMETER:

C** NGL .....ANZAHL GLFICHUNGEN

C** A. NK*NZ+2*( $(N K-2) / N Z+2) * N B * N Z+N B$ LANG.

C** NK....... HALAE AANDWETTE DER KOEFFIZENTENMATRTX

C. NZ.......ANZAHL ZEILEN PRO BLOCK ( NZ \& NGL).

C* B........AREFITSZONE DES SPEICHERS. MINDESTENS NB*NZ LANG NB.......ANZAHL BELASTUNGSFAELLE ( NB>O)

NTA...... IDENTIFIKATIONSNIMMER DER KOEFFIZENTFNMATRIX [A]

NTR...... IDENTIFIKATIONSNIMMER DER BELASTUNGSMATRIX [B]

NTX....... IDENTIFIKATIONSNUMMER DER LOESUNGSMATRIX $[X]$

NTR...... IDENTIFIKATIONSNUMMER DER RESIDUENMATRIX [R]

RLAENGE..RELATIVE LAENGE DER RESIDUENVEKTOREN.

$C * *$ 
Dieser Bericht beschreibt einige Computer-Programme, welche fur die elastische Analyse von Flächentragwerken nach der Methode der finiten Elemente benutzt werden können. Die FORTRAN IV Subroutinen DRPL21, DRPL18, DRSC18, VKPL28, VKPL 24 und VKSC24 berechnen die Steifigkeitsmatrizen sowie verschiedene Lastvektoren fur dreieckige und viereckige Platten- und Scheibenelemente. Die Subroutinen BANDMAT und BMRESID dienen zur Lðsung von grossen Gleichungssystemen, deren Koeffizentenmatrix eine Bandstruktur aufweist. Der erste Teil dieses Berichtes enthalt die Programmeschreibung. Im zweiten Teil werden die FORTRAN-Programme selbst angegeben.

SUMMARY

This report describes some computer programs which can be used for the elastic analysis of plates and shells by the finite element method. The FORTRAN IV subroutines DRPL21, DRPL18, DRSC18, VKPL28, VKPL24, and VKSC24 determine the stiffness matrices as we11 as several loading vectors for triangular and quadrilateral plate-bending and plate-streching elements. The subroutines BANDMAT and BMRESID are used for the solution of large symmetric systems of linear equations having a banded coefficient matrix. The first part of this report gives a short description of the different programs. The second part contains the program listings. 